\title{
Cahn-Hilliard theory for unstable granular fluids
}

\author{
T. P .C. van Noije and M. H. Ernst \\ Instituut voor Theoretische Fysica, Universiteit Utrecht, Postbus 80006, 3508 TA Utrecht, The Netherlands
}

(Received 1 July 1999)

\begin{abstract}
A Cahn-Hilliard-type theory for hydrodynamic fluctuations is proposed that gives a quantitative description of the slowly evolving spatial correlations and structures in density and flow fields in the early stages of evolution of freely cooling granular fluids. Two mechanisms for pattern selection and structure formation are identified: unstable modes leading to density clustering (mechanismlike spinodal decomposition, or " uplifting" in structural geology), and selective noise reduction (mechanismlike peneplanation in structural geology) leading to vortex patterns. As time increases, the structure factor for the density field develops a maximum, which shifts to smaller wave numbers. This corresponds to an approximately diffusively growing length scale for density clusters. Analytic expressions are derived for spatial correlation functions and structure factors that agree well with molecular dynamics simulations of a fluid of inelastic hard disks.
\end{abstract}

PACS number(s): 45.70.Qj, 47.20.-k, 05.40.-a

\section{INTRODUCTION}

Recently we have proposed a mesoscopic theory, based on fluctuating hydrodynamics with unstable modes (CahnHilliard-type theory) in order to calculate the structure factors and spatial correlations in driven and in freely cooling granular fluids. This theory permits a quantitative comparison with molecular dynamics (MD) simulations on fluids of inelastic hard spheres, over rather long-time intervals. Preliminary accounts of the results were published in Refs. [1,2] and documented in unpublished reports $[3,4]$. The basic goal of this paper is to show how the theory of fluctuating hydrodynamics or Langevin fluids [5] can be used in the field of dissipative systems, such as granular fluids, to give detailed theoretical predictions about the form of structure functions, about the nature of long- and short-range correlations, and to identify in granular fluids two different mechanisms for pattern formation: for density clusters an analog of spinodal decomposition [5] and for vortices an analog of peneplanation as occurring in structural geology [6]. Fluctuating hydrodynamics is one of the few available methods in statistical mechanics to calculate spatial correlations, and it is totally complementary to Boltzmann- or Enskog-type kinetic equations, which are based on the fundamental assumption of molecular chaos, i.e., on the absence of spatial correlations.

In fact, we have already applied the method to a randomly driven or heated inelastic hard sphere (IHS) fluid [7]. In that system the spatial correlations are very long ranged, $\sim r^{2-d}$, and are created by external noise that violates momentum conservation [8]. The same mechanism is creating the longranged $\sim r^{2-d}$ correlations between the height correlations in the Edwards-Wilkinson model for surface growth [9]. In the present model of the freely evolving IHS fluid the spatial correlations are much shorter ranged. For instance, in the incompressible limit they are proportional to $\sim r^{-d}$ [1]. So, the external driving drastically changes the nature of the spatial correlations.

The inelasticity of the collisions between grains makes driven and undriven granular fluids behave very differently from atomic or molecular fluids. A dramatic difference with elastic fluids is that granular fluids lose kinetic energy through inelastic collisions and cool if energy is not supplied externally. In a thermodynamic sense, granular fluids should be considered as "open" systems with an energy sink, created by the inelastic collisions. This collisional dissipation mechanism introduces several new time and length scales, which are often related to instabilities. In this paper we will focus on spatial correlation functions and structure factors, and on the underlying instabilities in freely evolving undriven granular fluids.

These instabilities have been studied by several authors using macroscopic or kinetic equations [10-18]. Goldhirsch and Zanetti [10] were the first to perform molecular dynamics (MD) simulations of an undriven two-dimensional (2D) system of smooth inelastic hard disks and observed the spontaneous formation of density clusters. The system is unstable against spatial density fluctuations, so inhomogeneities in the density field (clusters) slowly grow to macroscopic size. However, before this happens, the granular fluid, prepared in a spatially homogeneous state, remains in a spatially homogeneous cooling state (HCS) with a slowly decreasing temperature. Gradually spatial inhomogeneities appear in the flow field (vortex patterns), and only much later density clusters are being observed. In Fig. 1 we show typical snapshots of the momentum field and the density field, as obtained in

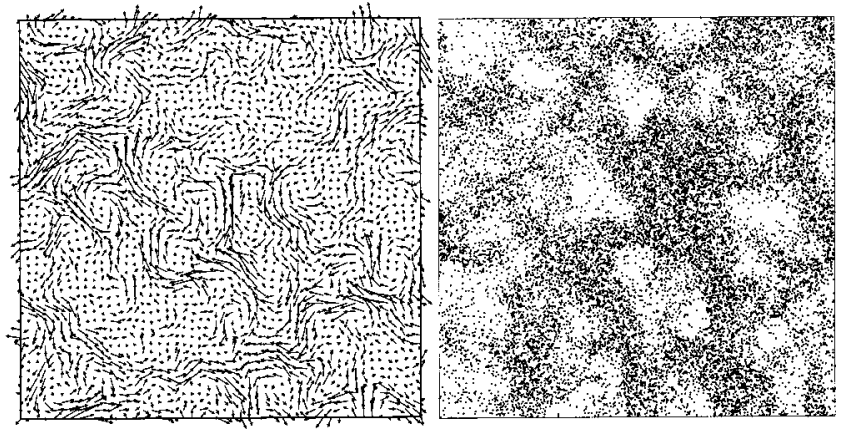

FIG. 1. Left: Velocity field after $\tau=80$ collisions per particle. The density is then still nearly homogeneous. Right: Density field at $\tau=160$. System of 50000 inelastic hard disks at a packing fraction $\phi=\frac{1}{4} \pi n \sigma^{2}=0.4$ and $\alpha=0.9$. 
MD simulations of a system of inelastic hard disks [1]. The flow field develops a large vorticity component and evolves into a "dense fluid of closely packed vortex structures," which is still homogeneous on scales large compared to the average vortex diameter $L_{\mathrm{V}}$, provided the system is sufficiently large. More detailed information from MD simulations on the late stages of evolution in clustering of granular fluids has recently been given in Ref. [18].

In order to understand and quantify the patterns of Fig. 1, we study the time-dependent spatial correlation functions of the corresponding fluctuations, using Landau and Lifshitz's theory of fluctuating hydrodynamics [19], adapted to dissipative hard sphere fluids, i.e., adapted to the presence of unstable modes. We present a theory that describes the buildup of spatial correlations in the density and flow field in the initial regime, where the inhomogeneities are governed by linear hydrodynamics, i.e., linearized around the homogeneous cooling state (HCS). To explain the theory we compare a freely evolving granular fluid with spinodal decomposition [5], where the observed phenomena are similar in several respects.

The observed instability and the concomitant pattern formation are explained by the Cahn-Hilliard $(\mathrm{CH})$ theory [5]. Similar theories have also been used for two-dimensional turbulence (see Frisch [20] and references therein), where the behavior of the fluctuations in the flow field of incompressible fluids has been described in terms of negative eddy viscosities. Vorticity modes with negative effective viscosities have also been used by Rothman to study vortex formation in lattice gas cellular automata [21].

The instability of undriven granular fluids also differs in many details from spinodal decomposition. The former is a slow process, the latter a fast process. Consequently, the Cahn-Hilliard theory in spinodal decomposition only describes the onset length and time scales of phase separation. As the formation of vortices and clusters in undriven granular fluids is a rather slow process, the present theory is expected to give a good description up to times which are rather large (see Figs. 1 and 2), provided the inelasticity and the density are not too large.

The most important function to describe the clustering instability are structure factors $S(k, t)$ in density and flow field. Goldhirsch et al. [11] have initiated the study of these structure factors, and related in a qualitative way the structure at small $k$ to the most unstable shear modes. They presented a nonlinear analysis to explain the enslaving of density fluctuations by the vorticity field. This analysis reveals that the length scale associated with the late stages of nonlinear clustering is of the order $\xi_{\perp} \sim l_{0} / \sqrt{\gamma_{0}}$, where $l_{0}$ is the mean free path. Brey et al. have also studied the nonlinear response of the density field to an initially excited $\mathbf{k}$ mode in the transverse flow field [22].

A first step in the theoretical understanding of $S_{n n}(k, t)$ for density fluctuations has been given by Deltour and Barrat [15]. These authors have shown how the growth rate of $S$ in the linear regime is determined by the most unstable longwavelength part of the heat mode, in which the density couples to longitudinal velocity perturbations.

In two preliminary publications $[1,2]$ we have only considered long-range correlations in the flow field to illustrate the results of fluctuating hydrodynamics. This theory yields

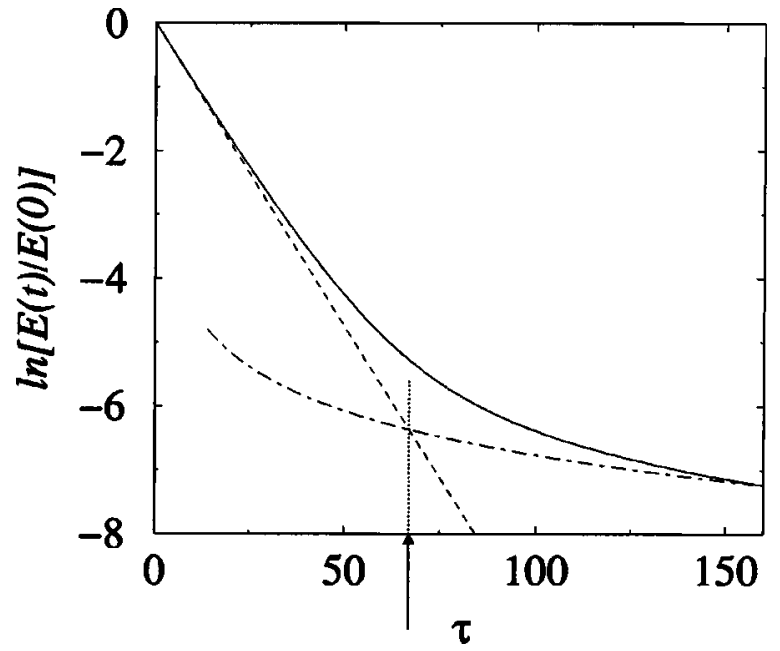

FIG. 2. Kinetic energy per particle $E$ versus number of collisions per particle $\tau$ for $\phi=0.4$ and $\alpha=0.9$. Initially $E$ is equal to the temperature $T_{0}$ and follows Haff's homogeneous cooling law (2). The arrow indicates a crossover time $\tau_{c} \simeq 67$ from the homogeneous cooling state to the nonlinear clustering regime. Then spatial inhomogeneities become important and slow down the energy decay. The dashed line represents Haff's law (2) and the dashed-dotted line the result of Ref. [17] for the long-time energy decay.

predictions, including long-range tails $\sim r^{-d}$ in $d$-dimensional fluids in the incompressible limit, that for nearly elastic particles $(\alpha \geqslant 0.9)$ agree well with twodimensional MD simulations up to large distances. As the transverse velocity fluctuations of an incompressible fluid do not couple to the density fluctuations in a linear theory, this theory gives no information on the structure factor $S_{n n}(k, t)$ for density fluctuations.

The plan of the paper is as follows. In Sec. II we briefly discuss the hydrodynamic equations, the decay of the total energy, and the properties of unstable shear and heat modes (with supporting technicalities in Appendix A). In Sec. III we construct the theory of fluctuating hydrodynamics for granular fluids, and present the general method on how to calculate structure factors and spatial correlations (with technicalities about Fourier and Bessel transformation in Appendix B). Section IV focuses on the flow field, and identifies the formation of vortex patterns, as peneplanation, while Sec. $\mathrm{V}$ deals with analogous properties for the density field, and identifies the density clustering as an analog of "spinodal decomposition" or "uplifting" to stay in a geological terminology. Both sections derive simple analytic approximations for structure factors and correlations valid for long times. We end with some conclusions in Sec. VI.

\section{HYDRODYNAMICS}

The macroscopic hydrodynamic equations for inelastic hard sphere (IHS) fluids are necessary to set the stage for describing fluctuations by Langevin-type equations. We assume that IHS hydrodynamics for weakly inelastic systems can be described by the standard hydrodynamic conservation equations supplemented by a sink term $\Gamma$ in the temperature balance equation [23], 


$$
\begin{gathered}
\partial_{t} n+\nabla \cdot(n \mathbf{u})=0, \\
\partial_{t} \mathbf{u}+\mathbf{u} \cdot \nabla \mathbf{u}=-\frac{1}{\rho} \nabla \cdot \boldsymbol{\Pi}, \\
\partial_{t} T+\mathbf{u} \cdot \boldsymbol{\nabla} T=-\frac{2}{d n}(\boldsymbol{\nabla} \cdot \mathbf{J}+\boldsymbol{\Pi}: \nabla \mathbf{u})-\Gamma .
\end{gathered}
$$

Moreover, $\rho=m n$ is the mass density, $\mathbf{u}$ the flow velocity, and $\frac{1}{2} d n T$ the kinetic energy density in the local rest frame of the IHS fluid and $d$ the number of dimensions. The pressure tensor $\Pi_{\alpha \beta}=p \delta_{\alpha \beta}+\delta \Pi_{\alpha \beta}$ contains the local pressure $p$ and the dissipative momentum flux $\delta \Pi_{\alpha \beta}$, which is proportional to $\nabla_{\alpha} u_{\beta}$ and contains the kinematic and longitudinal viscosities $\nu$ and $\nu_{l}$. The constitutive relation for the heat flux, $\mathbf{J}=-\kappa \boldsymbol{\nabla} T$, defines the heat conductivity $\kappa$. The quantity $\Gamma$ is the average rate of energy loss through collisional dissipation, which distinguishes Eqs. (1) from those of an elastic fluid. The above equations can be justified to lowest order in the inelasticity [24,25], and will be used for weakly inelastic systems, where thermodynamic and transport properties are assumed to be given by those of elastic hard sphere fluids (see Appendix A).

The energy balance equation for the heated fluid in Ref. [7], as opposed to the freely evolving fluid discussed here, contains apart from the energy sink, $-\Gamma$, also an energy source term. It is, however, not this inconspicuous mean energy source in the macroscopic energy balance that is responsible for the large difference in spatial correlations between driven and undriven granular fluids, but the noise characteristics of the energy source.

Kinetic theory provides an exact expression for the collisional dissipation rate $\Gamma$. It can be derived from the microscopic energy loss per collision. An explicit derivation can be found in Refs. [26,27]. For the present purpose, however, a phenomenological derivation suffices, which proceeds as follows. On average, a particle loses per collision an amount $\sim \gamma_{0} T$ of its kinetic energy, and per unit time an amount $\sim \gamma_{0} \omega T$, where $\gamma_{0} \equiv\left(1-\alpha^{2}\right) / 2 d$ is the degree of inelasticity. It is determined by the coefficient $\alpha$ of normal restitution, which defines the inelastic hard sphere collisions [23]. Here $\omega$ is the average collision frequency [28], given by Enskog's theory for dense hard sphere fluids [28], and quoted in Appendix A. It is proportional to $\sqrt{T}$. This argument gives (apart from a numerical factor) $\Gamma=2 \gamma_{0} \omega T$.

For an understanding of what follows two properties of undriven granular fluids are important: (i) the existence of a homogeneous cooling state (HCS) and (ii) its linear instability against spatial fluctuations. The hydrodynamic equations for an IHS fluid, initialized in a homogeneous state with temperature $T_{0}$, admit a HCS solution with a homogeneous density $n$, a vanishing flow field, and a homogeneous temperature $T(t)$, determined by $\partial_{t} T=-\Gamma$. To solve this equation it is convenient to change to a new time variable, defined as $d \tau=\omega(T(t)) d t$, yielding $T(t)=T_{0} \exp \left(-2 \gamma_{0} \tau\right)$. To find the relation between the "internal" time $\tau$, which measures the average number of collisions suffered per particle within a time $t$, and the "external" time $t$, we integrate the relation for $d \tau$ using $\omega \sim \sqrt{T}$, with the result $\gamma_{0} \tau=\ln [1$ $\left.+\gamma_{0} t / t_{0}\right]$. In the elastic limit $\left(\gamma_{0} \rightarrow 0\right)$, it is proportional to the external time, $\tau=t / t_{0}$, measured in units of the mean free time $t_{0}=1 / \omega\left(T_{0}\right)$ at the initial temperature. The initial slope of $\tau(t)$ corresponds to the collision frequency $\omega\left(T_{0}\right)$ in the equilibrium state at $\tau=0$. A combination of these results yields the slow decay of the temperature,

$$
T(t)=T_{0} \exp \left(-2 \gamma_{0} \tau\right)=T_{0} /\left(1+\gamma_{0} t / t_{0}\right)^{2},
$$

as first derived by Haff [23]. The relation introduces a new intermediate (mesoscopic) time scale, the homogeneous cooling time $t_{e} \equiv t_{0} / \gamma_{0}$.

For small inelasticity and a wide range of densities the validity of Haff's law and the existence of the HCS has been verified by MD simulations $[10-15,18]$. The relation for the temperature or energy decay remains valid until a crossover time $\tau_{c}$ (defined in Fig. 2) to a diffusive regime, where the system remains spatially homogeneous, but where velocity fluctuations drive the system away from the HCS, and the decay of the total energy slows down to $\tau^{-d / 2}$ [17]. For still larger times and/or larger inelasticities different forms of algebraic energy decay, like $E \sim t^{-d / 2}$ [29] and $E \sim t^{-2 d /[d+2]}$ [30] have been proposed. The HCS solution becomes linearly unstable as soon as the length $L$ of the system exceeds some dynamic correlation length $\sim l_{0} / \sqrt{\gamma_{0}}$ [10-15], where $l_{0}$ is the (time-independent) mean free path. It is given by $l_{0}=\mathrm{v}_{0} / \omega$, where $\mathrm{v}_{0}(t)=\sqrt{2 T(t) / m}$ is the thermal velocity.

In general the HCS is highly nontrivial, as it exhibits correlations between the velocities and positions of different particles. In the "lowest order" description (for more refined approximations see $[26,27,31,32])$ the HCS corresponds to an equilibrium state, which is cooling adiabatically, i.e., with a time-dependent temperature (2). Here velocity correlations between different particles are absent, and position correlations are taken only into account through the pair correlation function at contact.

In the present paper we are interested in the buildup of correlations between spatial fluctuations in a system that is prepared in a homogeneous state at an initial temperature $T_{0}$. It reaches the HCS within a few mean free times $t_{0}$. Therefore, we can linearize Eqs. (1) around the homogeneous density $n$ and temperature $T(t)=T_{0} /\left[1+\gamma_{0} t / t_{0}\right]^{2}$, and the vanishing flow field of the slowly evolving HCS. The resulting set of linearized hydrodynamic equations, given in Eq. (A1) of Appendix A, contains the Enskog hard sphere transport coefficients, $\nu, \nu_{l}$, and $\kappa$, which are proportional to $\sqrt{T(t)}$, and depend therefore explicitly on time. It is again convenient to make the transformation $\mathrm{d} \tau=\omega \mathrm{d} t$, and introduce the rescaled variables $\delta \tilde{n}(\mathbf{r}, \tau)=\delta n(\mathbf{r}, t) / n, \quad \tilde{\mathbf{u}}(\mathbf{r}, \tau)$ $=\mathbf{u}(\mathbf{r}, t) / \mathrm{v}_{0}(t)$, and $\delta \widetilde{T}(\mathbf{r}, \tau)=\delta T(\mathbf{r}, t) / T(t)$. In these variables the equations of change for the macroscopic Fourier modes $\delta \tilde{n}(\mathbf{k}, \tau), \tilde{\mathbf{u}}(\mathbf{k}, \tau)$, and $\delta \widetilde{T}(\mathbf{k}, \tau)$, defined through $\delta \tilde{a}(\mathbf{k}, \tau)=\int d \mathbf{r} \exp (-i \mathbf{k} \cdot \mathbf{r}) \delta \tilde{a}(\mathbf{r}, \tau)$, become ordinary differential equations with time-independent coefficients. In matrix representation we write the above equations as

$$
\frac{\partial}{\partial \tau} \tilde{\delta \mathbf{a}}(\mathbf{k}, \tau)=\mathbf{M}(\mathbf{k}) \tilde{\delta \mathbf{a}}(\mathbf{k}, \tau)
$$

where components of $\tilde{\mathbf{a}}$ and $\mathbf{M}$ are labeled with $\{n, T, l, \perp\}$, and are explicitly given in Appendix A. The subscript $\perp$ in 


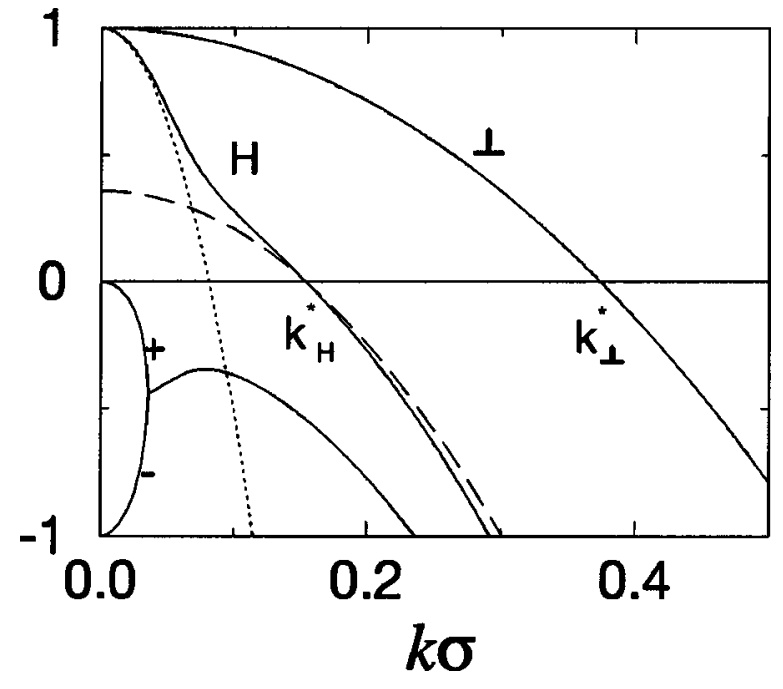

FIG. 3. Growth rates $\zeta_{\lambda} / \gamma_{0}$ for shear $(\lambda=\perp)$, heat $(\lambda=H)$, and sound $\left(\lambda= \pm\right.$ ) modes (nonpropagating for $k \sigma \ll \gamma_{0}$ ) versus $k \sigma$ for inelastic hard disks with $\alpha=0.9$ at a packing fraction $\phi=0.4\left(l_{0}\right.$ $\simeq 0.34 \sigma)$. The shear and heat mode are unstable for $k<k_{\perp}^{*}$ and $k$ $<k_{H}^{*}$, respectively.

the equation for $\tilde{u}_{\perp}$ refers to any of the $d-1$ directions perpendicular to $\mathbf{k}$, and the subscript $l$ denotes the longitudinal direction along $\mathbf{k}$. The validity of the hydrodynamic Eqs. (3) is restricted to wave numbers $k \ll 2 \pi / l_{0}$ to guarantee separation of kinetic and hydrodynamic scales, and to $k \ll 2 \pi / \sigma$, where $\sigma$ is the diameter of a disk or sphere, to guarantee that the Euler equations involve only local hydrodynamics. Already at moderate packing fractions of about $20 \%$ the mean free path for hard disks is less than one diameter, and the system starts to show nonlocal effects in the thermodynamics and transport properties for wavelengths $\lambda$, satisfying $l_{0}<\lambda$ $<\sigma$.

Necessary ingredients in our subsequent analysis are the eigenvalues or dispersion relations $\zeta_{\lambda}(k)$ and corresponding eigenvectors of $\mathbf{M}$, which are given in Appendix A. Dispersion relations $\zeta_{\lambda}(k)$ for the IHS fluid have been calculated, for instance, in Ref. [15]. Typical results of our calculations are shown in Fig. 3. The most striking feature is that there are two eigenvalues, $\zeta_{\perp}$ and $\zeta_{H}$, that are positive for $k$ below the stability thresholds $k_{\perp}^{*}$ and $k_{H}^{*}$, i.e., two linearly unstable modes with exponential growth rates. In the dissipative range [12] $\left(k l_{0} \ll \gamma_{0}\right)$ all eigenvalues are real; propagating modes are absent. Around $k l_{0} \sim \mathcal{O}\left(\gamma_{0}\right)$, two eigenvalues become complex conjugates and the corresponding (sound) modes become propagating, with a (rescaled) propagation speed $c_{s}$, which equals the adiabatic sound speed in elastic hard sphere fluid, to lowest order in $\gamma_{0}$. In the normal or elastic range $\left(k l_{0} \propto \sqrt{\gamma_{0}}\right)$, heat conduction, which is $\mathcal{O}\left(k^{2} l_{0}^{2}\right)$, becomes dominant when $\gamma_{0}$ is assumed to be sufficiently small so that $\gamma_{0} \ll \sqrt{\gamma_{0}}$. In the latter range, the dispersion relations and eigenmodes resemble those of an elastic fluid.

The most simple modes are the $(d-1)$-fold degenerate transverse velocity or shear modes, which are decoupled from the remaining modes, and given by $\tilde{\mathbf{u}}_{\perp}(\mathbf{k}, \tau)$ $=\tilde{\mathbf{u}}_{\perp}(\mathbf{k}, 0) \exp \left[\zeta_{\perp}(k) \tau\right]$, where $\zeta_{\perp}(k)=\gamma_{0}\left(1-k^{2} \xi_{\perp}^{2}\right)$. We point out for later reference that all correlation lengths $\xi_{a}$ used in this paper are defined in Eqs. (A3) and (A4) of Ap-

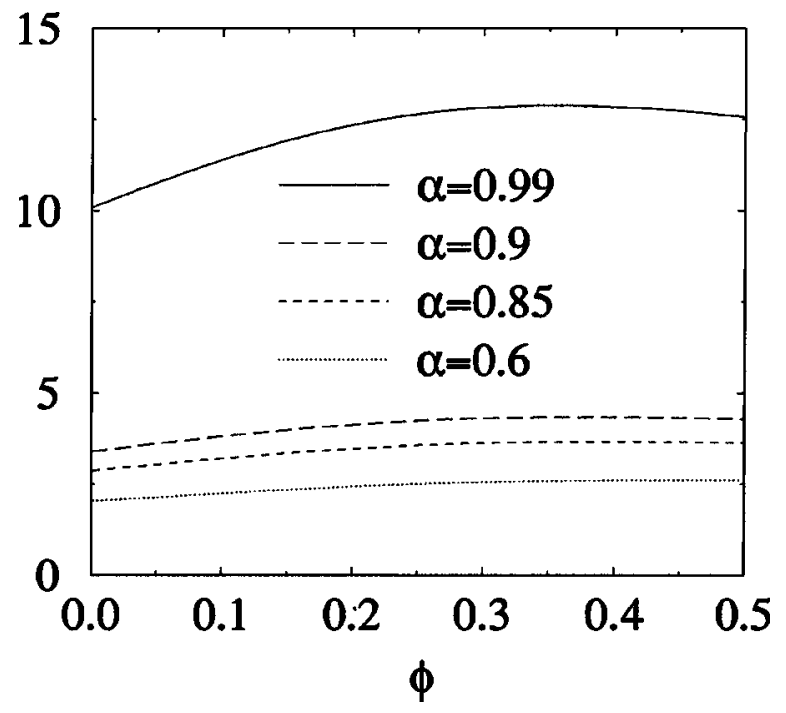

FIG. 4. Ratio $\xi_{\|} / \xi_{\perp}$ versus packing fraction $\phi$ of inelastic disks, with definitions of $\xi$ 's in Eqs. (A3) and (A4).

pendix A. The shear mode is unstable for $k<k_{\perp}^{*}=1 / \xi_{\perp}$.

There is another unstable mode, referred to as "heat mode," that is essential for explaining the formation of vortices and density clusters. The corresponding eigenvalue $\zeta_{H}(k)$, the root of a cubic equation, is shown in Fig. 3. It is unstable for $\zeta_{H}>0$, i.e., for $k<k_{H}^{*}$, with $k_{H}^{*}$ calculated in Eq. (A7). It has simple limiting behavior,

$$
\begin{aligned}
\zeta_{H}(k) & \simeq \gamma_{0}\left(1-k^{2} \xi_{\|}^{2}\right) \quad\left(k l_{0} \ll \gamma_{0}\right) \\
& \simeq \gamma_{0}\left(\zeta_{0}-k^{2} \xi_{H}^{2}\right) \quad\left(k l_{0} \propto \sqrt{\gamma_{0}}\right),
\end{aligned}
$$

as derived later in Eqs. (A7) and (A10), and shown in Fig. 3, respectively, as a dashed and a dotted line. In the dissipative range the eigenmode is a purely longitudinal velocity fluctuation. In the elastic range around $k_{H}^{*}\left(k \propto \sqrt{\gamma_{0}}\right)$ the eigenmode is to dominant order in $\gamma_{0}$ the heat mode of an elastic hard sphere fluid. Furthermore, we point out that $\xi_{\perp}$ and $\xi_{H}$ diverges as $1 / \sqrt{\gamma_{0}}$ for small $\gamma_{0}$, while $\xi_{\|} \sim 1 / \gamma_{0}$. As a consequence the correlation lengths $\xi_{\perp}$ and $\xi_{H}$ are well separated from $\xi_{\|}$for small inelasticity, as shown in Fig. 4. The sound modes will not be needed.

We also observe that the instability of the shear and heat modes is a long-wavelength instability. As a consequence effects of the boundaries are important for finite systems, and the various instabilities are suppressed in small systems. When using periodic boundary conditions, the instabilities are suppressed if $k_{\min }=2 \pi / L$ is larger than $k_{\perp}^{*}$ or $k_{H}^{*}$. When decreasing the system length $L=V^{1 / d}$ at fixed inelasticity, first the heat mode will become stable $\left(k_{H}^{*}<k_{\min }<k_{\perp}^{*}\right)$. In this range the density (coupled to the heat mode) is linearly stable, and density inhomogeneities can only be created via a nonlinear coupling to the unstable shear mode $[10,22]$. Decreasing the system size even further $\left(k_{\perp}^{*}<k_{\min }\right)$ will stabilize the shear mode and thus the HCS itself. In Sec. III we present a mesoscopic theory to describe the dynamics of the long-wavelength fluctuations in the system. 


\section{MESOSCOPIC HYDRODYNAMICS}

\section{A. Langevin equations}

In this section we construct the general theory of fluctuating hydrodynamics for granular fluids, and point out the differences between the applications of this theory to freely evolving and to randomly driven systems. The quantities to be calculated are the equal-time spatial correlation functions of the fluctuating hydrodynamic fields $\delta a(\mathbf{r}, t)=a(\mathbf{r}, t)$ $-\langle a\rangle$, where $a, b=\left\{n, T, u_{\alpha}\right\}$ with $\alpha=x, y, \ldots$ denoting Cartesian components,

$$
G_{a b}(\mathbf{r}, t)=V^{-1} \int d \mathbf{r}^{\prime}\left\langle\delta a\left(\mathbf{r}+\mathbf{r}^{\prime}, t\right) \delta b\left(\mathbf{r}^{\prime}, t\right)\right\rangle .
$$

The structure factors are the corresponding Fourier transforms,

$$
\begin{aligned}
S_{a b}(\mathbf{k}, t) & =\int d \mathbf{r} \exp (-i \mathbf{k} \cdot \mathbf{r}) G_{a b}(\mathbf{r}, t) \\
& =V^{-1}\langle\delta a(\mathbf{k}, t) \delta b(-\mathbf{k}, t)\rangle,
\end{aligned}
$$

where $\delta a(\mathbf{k}, t)$ is the Fourier transform of $\delta a(\mathbf{r}, t)$, and $V$ $=L^{d}$ is the volume of the system. We study the spatial fluctuations $\delta a(\mathbf{r}, t)$ of the hydrodynamic fields around a reference state, which leads to a Cahn-Hilliard-type theory [5] for the structure factors. The dynamics of these fluctuations can be described by the fluctuating hydrodynamic equations [19], obtained from the macroscopic hydrodynamic equations (3) by adding noise sources to the momentum and energy balance equations. The noise sources are denoted by $\boldsymbol{\nabla} \cdot \hat{\boldsymbol{\Pi}}$ and $\boldsymbol{\nabla} \cdot \hat{\mathbf{J}}$, respectively, which conserve momentum and energy. The currents $\hat{\mathbf{I}}$ and $\hat{\mathbf{J}}$ are considered as Gaussian white noise, local in space, and their correlations are determined by some appropriately formulated fluctuation-dissipation theorem for the reference state.

In elastic fluids the reference state would be the thermal equilibrium state. In driven systems it would be a nonequilibrium steady state (NESS). In the present case the reference state is the slowly evolving homogeneous cooling state. The same reference state has been used by Goldhirsch and one of the authors $[3,32]$ to derive Green-Kubo formulas for transport coefficients in the undriven IHS fluid. In the lowest approximation [27] it may be considered as an adiabatically changing equilibrium state with a constant density, a vanishing flow field, and a time-dependent temperature, described by Haff's law (2). The basic extension required for application to IHS fluids is the assumption that the fluctuationdissipation theorem also applies to the HCS with an adiabatically changing temperature $T(t)$. This assumption relates the noise strengths to the transport coefficients through [19]

$$
\begin{aligned}
\left\langle\hat{\Pi}_{\alpha \beta}(\mathbf{r}, t) \hat{\Pi}_{\gamma \delta}\left(\mathbf{r}^{\prime}, t^{\prime}\right)\right\rangle= & 2 \rho T\left[\nu\left(\delta_{\alpha \gamma} \delta_{\beta \delta}+\delta_{\alpha \delta} \delta_{\beta \gamma}\right)\right. \\
& \left.+\left(\nu_{l}-2 \nu\right) \delta_{\alpha \beta} \delta_{\gamma \delta}\right] \delta\left(\mathbf{r}-\mathbf{r}^{\prime}\right) \delta \\
& \left.\times\left(t-t^{\prime}\right)\right],
\end{aligned}
$$

where the total coefficient in front of $\nu$ is a traceless isotropic tensor of rank 4. For dimensional reasons, the transport coefficients in systems with hard sphere type interactions, like IHS, are proportional to $\sqrt{T(t)}$.

In the present theory for nearly elastic fluids we linearize the nonlinear Langevin equations, obtained from Eq. (1), around the HCS. By applying the transformations introduced above Eqs. (3) we obtain the dynamic equations for the rescaled variables, in the standard form of a set of Langevin equations with constant coefficients,

$$
\frac{\partial}{\partial \tau} \tilde{\delta \mathbf{a}}(\mathbf{k}, \tau)=\mathbf{M}(\mathbf{k}) \tilde{\delta \mathbf{a}}(\mathbf{k}, \tau)+\hat{\mathbf{f}}(\mathbf{k}, \tau)
$$

where the hydrodynamic matrix $\mathbf{M}(\mathbf{k})$ represents the deterministic part of the Langevin equation, and $\hat{\mathbf{f}}$ represents the rescaled internal fluctuations in the momentum and heat flux, which are characterized as Gaussian white noise by Eq. (7).

The equation of motion for the matrix of rescaled structure factors, $\widetilde{S}_{a b}(\mathbf{k}, \tau)=V^{-1}\langle\delta \tilde{a}(\mathbf{k}, \tau) \delta \widetilde{b}(-\mathbf{k}, \tau)\rangle$, can now be derived from Eq. (8), and yields

$$
\frac{\partial \widetilde{\mathbf{S}}(\mathbf{k}, \tau)}{\partial \tau}=\mathbf{M}(\mathbf{k}) \cdot \widetilde{\mathbf{S}}(\mathbf{k}, \tau)+\widetilde{\mathbf{S}}(\mathbf{k}, \tau) \cdot \mathbf{M}^{T}(-\mathbf{k})+\mathbf{C}(k),
$$

where $\mathbf{M}^{T}$ is the transpose of $\mathbf{M}$. It is to be solved for given initial values $\widetilde{\mathbf{S}}(\mathbf{k}, 0)$, referring to the state in which the system has been prepared at the initial state $t=0$.

In terms of the rescaled variables the Gaussian white noise (7) has the standard from with constant coefficients, given by the covariance matrix, $\mathbf{C}(k)$, with

$$
V^{-1}\left\langle\hat{f}_{a}(\mathbf{k}, \tau) \hat{f}_{b}\left(-\mathbf{k}, \tau^{\prime}\right)\right\rangle=C_{a b}(k) \delta\left(\tau-\tau^{\prime}\right) .
$$

It is diagonal with nonvanishing elements

$$
\begin{gathered}
C_{T T}=8 \kappa k^{2} / d^{2} n^{2} \omega=4 \gamma_{0} k^{2} \xi_{T}^{2} / d n, \\
C_{l l}=\nu_{l} k^{2} / n \omega=\gamma_{0} k^{2} \xi_{l}^{2} / n, \\
C_{\perp \perp}=\nu k^{2} / n \omega=\gamma_{0} k^{2} \xi_{\perp}^{2} / n,
\end{gathered}
$$

where the elements $(T T),(l l)$, and $(\perp \perp)$ follow directly from Eqs. (7) and the definitions of the correlation lengths in Eqs. (A3) by considering $i k \hat{J}_{l}, 2 i k \hat{\Pi}_{l l} / \rho \omega$, and $2 i k \hat{\Pi}_{l \perp} / \rho \omega$, respectively, and using the relation $\delta(\tau)$ $=\delta(t) / \omega(T(t))$.

Here it is of interest to point out that the internal Langevin noise, $i \mathbf{k} \cdot \hat{\boldsymbol{\Pi}}$ and $i \mathbf{k} \cdot \hat{\mathbf{J}}$, in the undriven IHS fluid conserves energy and momentum. This is the essential difference with the randomly driven IHS fluid of Ref. [7], where the external noise does not conserve momentum and energy, i.e., the noise sources are not proportional to $k$ at small $k$. Consequently, the noise strengths corresponding to $\mathbf{C}$ are missing the factors $k^{2}$ in Eqs. (11), which in turn lead to much longer spatial correlations. For a more extensive discussion on the difference between Langevin noise that does or does not conserve the macroscopic conservation laws we refer to Grinstein et al. [8]. 
The formal solution of Eq. (9) for the matrix of rescaled structure factors is then

$$
\begin{aligned}
\widetilde{\mathbf{S}}(\mathbf{k}, \tau)= & \exp [\mathbf{M}(\mathbf{k}) \tau] \cdot \widetilde{\mathbf{S}}(\mathbf{k}, 0) \cdot \exp \left[\mathbf{M}^{T}(-\mathbf{k}) \tau\right] \\
& +\int_{0}^{\tau} d \tau^{\prime} \exp \left[\mathbf{M}(\mathbf{k}) \tau^{\prime}\right] \cdot \widetilde{\mathbf{C}}(k) \cdot \exp \left[\mathbf{M}^{T}(-\mathbf{k}) \tau^{\prime}\right]
\end{aligned}
$$

At the initial time $(\tau=0)$ the system is prepared in a thermal equilibrium state of elastic hard spheres with density $n$ and temperature $T_{0}$. Consequently, all elements of $\widetilde{\mathbf{S}}(\mathbf{k}, 0)$ are known. Moreover, the evolution equations (9) and (3) are only valid for $k \ll\left\{2 \pi / l_{0}, 2 \pi / \sigma\right\}$. So the initial values $\widetilde{\mathbf{S}}(\mathbf{k}, 0)$ are only needed for $k \sigma \ll 2 \pi$, where they are given by their limiting values as $k \rightarrow 0$. [Note that $\mathbf{k}$ should not be set equal to $\mathbf{0}$, because $\delta \tilde{n}(\mathbf{0}, t)=0$ when the total number of particles is fixed.] The nonvanishing $\widetilde{S}_{a b}(\mathbf{k}, 0)$ with $a, b=\{n, T, l, \perp\}$ are given by the equipartition values for elastic hard sphere fluids in thermal equilibrium,

$$
\begin{gathered}
\widetilde{S}_{n n}(k, 0)=(T / n)(\partial n / \partial p)_{T} \equiv 1 /\left[2 n c_{T}^{2}\right], \\
\widetilde{S}_{T T}(k, 0)=2 /[d n], \\
\widetilde{S}_{l l}(k, 0)=\widetilde{S}_{\perp \perp}(k, 0)=1 /[2 n],
\end{gathered}
$$

where the first relation is the Ornstein-Zernike relation for density fluctuations.

\section{B. Spectral analysis}

To gain insight in the mechanisms that developed structure and correlations over distances much larger than the particle diameter $\sigma$, a spectral analysis is required. Numerical solutions, which will be discussed in a later subsection, do not provide much insight, but are only needed for a detailed quantitative comparison of the present theory with MD simulations. For a theoretical analysis it is more convenient to study the deviations from the initial values in thermal equilibrium, defined as the excess structure factor,

$$
\widetilde{\mathbf{S}}^{+}(\mathbf{k}, \tau)=\widetilde{\mathbf{S}}(\mathbf{k}, \tau)-\widetilde{\mathbf{S}}(\mathbf{k}, 0) .
$$

The reason is that $\widetilde{\mathbf{S}}(\mathbf{k}, \tau)$ at fixed $\tau$ approaches $\widetilde{\mathbf{S}}(\mathbf{k}, 0)$ when $k$ increases. Consequently, $\widetilde{\mathbf{S}}^{+}(\mathbf{k}, \tau)$ is only sizable for small $k$, and therefore more suitable for perturbative treatment based on hydrodynamics. This can be understood by inspection of the hydrodynamic matrix $\mathbf{M}(\mathbf{k})$ for the IHS fluid, given in Appendix A. For $k l_{0} \gg \sqrt{\gamma_{0}}$ all terms of $\mathcal{O}\left(\gamma_{0}\right)$ can be neglected, and the hydrodynamic matrix reduces to the elastic one, $\mathbf{E}(\mathbf{k})$, and Eq. (9) reduces to

$$
\mathbf{E}(\mathbf{k}) \cdot \widetilde{\mathbf{S}}(\mathbf{k}, 0)+\widetilde{\mathbf{S}}(\mathbf{k}, 0) \cdot \mathbf{E}^{T}(-\mathbf{k})+\mathbf{C}(k)=\mathbf{0},
$$

as can be verified using Eqs. (11) and (13). The relation above is in fact equivalent to the fluctuation-dissipation theorem (7) for elastic hard spheres in rescaled units.

Subtracting this equation from Eq. (9) yields

$$
\frac{\partial}{\partial \tau} \widetilde{\mathbf{S}}^{+}(\mathbf{k}, \tau)=\mathbf{M}(\mathbf{k}) \cdot \widetilde{\mathbf{S}}^{+}(\mathbf{k}, \tau)+\widetilde{\mathbf{S}}^{+}(\mathbf{k}, \tau) \cdot \mathbf{M}^{T}(-\mathbf{k})+\mathbf{B}(k)
$$

After some rearrangements the source term is found as

$$
\begin{aligned}
\mathbf{B}(k)= & {[\mathbf{M}(\mathbf{k})-\mathbf{E}(\mathbf{k})] \cdot \widetilde{\mathbf{S}}(\mathbf{k}, 0)+\widetilde{\mathbf{S}}(\mathbf{k}, 0) } \\
& \times\left[\mathbf{M}^{T}(-\mathbf{k})-\mathbf{E}^{T}(-\mathbf{k})\right] .
\end{aligned}
$$

Its nonvanishing matrix elements are

$$
\begin{gathered}
B_{n T}=B_{T n}=-\gamma_{0} a \widetilde{S}_{n n}(k, 0), \\
B_{T T}=-2 \gamma_{0} \widetilde{S}_{T T}(k, 0), \\
B_{l l}=2 \gamma_{0} \widetilde{S}_{l l}(k, 0), \\
B_{\perp \perp}=2 \gamma_{0} \widetilde{S}_{\perp \perp}(k, 0),
\end{gathered}
$$

where $a$ is defined in Eqs. (A5). The formal solution of Eq. (16) with the initial value $\widetilde{\mathbf{S}}^{+}(\mathbf{k}, 0)=0$ becomes

$$
\widetilde{\mathbf{S}}^{+}(\mathbf{k}, \tau)=\int_{0}^{\tau} d \tau^{\prime} e^{\mathbf{M}(\mathbf{k}) \tau^{\prime}} \cdot \mathbf{B}(k) \cdot e^{\mathbf{M}^{T}(-\mathbf{k}) \tau^{\prime}} .
$$

The spectral decomposition (A6) of $\mathbf{M}$ allows us to write the components $a, b=\{n, T, l, \perp\}$ of Eq. (19) as

$$
\widetilde{S}_{a b}^{+}(\mathbf{k}, \tau)=\sum_{\lambda \mu} \mathcal{B}_{a b}^{\lambda \mu}(k)\left(\frac{\exp \left[\left(\zeta_{\lambda}+\zeta_{\mu}\right) \tau\right]-1}{\zeta_{\lambda}+\zeta_{\mu}}\right),
$$

where $\lambda, \mu$ label the hydrodynamic modes and

$$
\mathcal{B}_{a b}^{\lambda \mu}(k)=w_{\lambda a}(\mathbf{k}) w_{\mu b}(-\mathbf{k})\left\langle\mathbf{v}_{\lambda}(\mathbf{k})|\mathbf{B}(k)| \mathbf{v}_{\mu}(-\mathbf{k})\right\rangle,
$$

where $\mathbf{v}_{\lambda a}$ and $\mathbf{v}_{\mu b}$ are contracted with $B_{a b}$. Once the eigenvalues $\zeta_{\lambda}(k)$ and eigenvectors $\mathbf{v}_{\lambda}, \mathbf{w}_{\lambda}$ are known, the structure factors can be calculated. The results (20) contain exponentially growing factors describing the unstable modes with $\zeta_{\lambda}>0$, as in the Cahn-Hilliard $(\mathrm{CH})$ theory for spinodal decomposition. The present theory includes in Eq. (8) Langevin noise terms, which guarantee that the fluctuations at large $k$ reach their thermal equipartition values, as the collisional dissipation can be neglected at large $k$. It is equivalent to the Cahn-Hilliard-Cook theory [5]. If the Langevin noise is neglected by setting $\widetilde{\mathbf{C}}(k)=\mathbf{0}$ in Eq. (12), we obtain the predictions of the noiseless $\mathrm{CH}$ theory,

$$
\widetilde{\mathbf{S}}(\mathbf{k}, \tau)=\exp [\mathbf{M}(\mathbf{k}) \tau] \cdot \widetilde{\mathbf{S}}(\mathbf{k}, 0) \cdot \exp \left[\mathbf{M}^{T}(-\mathbf{k}) \tau\right],
$$

or in component form

$$
\widetilde{S}_{a b}(\mathbf{k}, \tau)=\sum_{\lambda \mu} \widetilde{\mathcal{S}}_{a b}^{\lambda \mu}(\mathbf{k}) \exp \left[\left(\zeta_{\lambda}+\zeta_{\mu}\right) \tau\right]
$$

where $\widetilde{\mathcal{S}}_{a b}^{\lambda \mu}(\mathbf{k})$ is defined by Eq. (21) with $\widetilde{\mathbf{B}}(k)$ replaced by $\widetilde{\mathbf{S}}(\mathbf{k}, 0)$. 
The explicit solutions can be studied using the eigenvalues and eigenfunctions in different ranges of wave numbers. For the very long wavelengths in the dissipative range $\left(k l_{0}\right.$ $\left.\ll \gamma_{0}\right)$ this can be done by $k$ expansion at fixed $\gamma_{0}$. The elastic or normal range $\left(k l_{0} \propto \sqrt{\gamma_{0}}\right)$ is accessible to analysis by rescaling the wavelengths as $k=\sqrt{\gamma_{0}} q$, considering $q$ $=\mathcal{O}(1)$, and taking the small- $\gamma_{0}$ limit subsequently, as discussed in Appendix A.

\section{Structure and correlations}

Suppose we have calculated the structure factors, what information can we extract from them? An elastic fluid in thermal equilibrium does not show any structure on hydrodynamic length scales $\left(k \leqq \min \left\{1 / l_{0}, 1 / \sigma\right\}\right)$. This means that the hydrodynamic structure factors $S_{a b}(k)$ are totally flat, independent of $k$, as can be seen in Eq. (13). The corresponding hydrodynamic correlation functions are short ranged, $G_{a b}(\mathbf{r}, t) \sim \delta(\mathbf{r})$, on these length scales. Development of structure on length scales above the microscopic scales $\left\{l_{0}, \sigma\right\}$ will manifest itself in the appearance of one or more maxima or peaks in the structure factors. A linear instability will manifest itself in a structure factor that grows exponentially in time. With these concepts in mind, we analyze the structure factors in Eq. (20) for the IHS fluid, as we want to determine which physical excitations are responsible for the features observed in the MD simulations and in the numerical solutions.

Once the structure factors have been obtained, the correlation functions can be calculated by Fourier inversion. When $a$ and $b$ refer to $n$ and $T$ the components of $S_{a b}(\mathbf{k}, t)$ and $G_{a b}(\mathbf{r}, t)$ are scalar isotropic functions only depending on $|\mathbf{k}|$ and $|\mathbf{r}|$, respectively. When $(a, b)=(\alpha, \beta)$ refer to Cartesian components $u_{\alpha}$ of the flow field, then $S_{\alpha \beta}(\mathbf{k}, t)$ is a second rank isotropic tensor field, which can be separated into two independent isotropic scalar functions:

$$
S_{\alpha \beta}(\mathbf{k}, t)=\hat{k}_{\alpha} \hat{k}_{\beta} S_{\|}(k, t)+\left(\delta_{\alpha \beta}-\hat{k}_{\alpha} \hat{k}_{\beta}\right) S_{\perp}(k, t),
$$

where $S_{\|}(k, t)$ and $S_{\perp}(k, t)$ are given by Eq. (6) with $\delta a$ and $\delta b$ equal to $u_{l}$ and $u_{\perp}$, respectively. A similar separation applies to the spatial correlation functions,

$$
\begin{aligned}
G_{\alpha \beta}(\mathbf{r}, t) & =V^{-1} \sum_{\mathbf{k}} \exp (i \mathbf{k} \cdot \mathbf{r}) S_{\alpha \beta}(\mathbf{k}, t) \\
& =\hat{r}_{\alpha} \hat{r}_{\beta} G_{\|}(r, t)+\left(\delta_{\alpha \beta}-\hat{r}_{\alpha} \hat{r}_{\beta}\right) G_{\perp}(r, t) .
\end{aligned}
$$

Here the scalar functions $G_{\|}(r, t)$ and $G_{\perp}(r, t)$ refer to the tensor components of $G_{\alpha \beta}(r, t)$, which are, respectively, parallel and perpendicular to the relative position $\mathbf{r}$. They do not represent the inverse transforms of the scalar functions $S_{\|}(k, t)$ and $S_{\perp}(k, t)$. Firstly we note that the Fourier series in Eq. (25) can be replaced by a Fourier integral, provided that the system is sufficiently large. Then, for periodic boundary conditions, as used in MD simulations, $V^{-1} \Sigma_{\mathbf{k}}$ can be replaced by $(2 \pi)^{-d} \int d \mathbf{k}$.

Secondly, the inverse Fourier transform $\mathbf{G}(\mathbf{r}, t)$ of $\mathbf{S}(\mathbf{k}, t)$ only exists as a classical function, if $\mathbf{S}^{\infty}(t) \equiv \lim _{k \rightarrow \infty} \mathbf{S}(\mathbf{k}, t)$ vanishes. If $\mathbf{S}(\mathbf{k}, t)$ approaches a nonvanishing constant $\mathbf{S}^{\infty}(t)$, it yields a distribution $\delta(\mathbf{r})$. So,

$$
\mathbf{G}(\mathbf{r}, t)=\mathbf{S}^{\infty} \delta(\mathbf{r})+\int \frac{d \mathbf{k}}{(2 \pi)^{d}} \exp (i \mathbf{k} \cdot \mathbf{r}) \mathbf{S}^{+}(\mathbf{k}, t) .
$$

Note that the limiting values $\widetilde{S}_{a b}^{\infty}$, when expressed in terms of the rescaled variables, are given by the time and (wave number) independent values $\widetilde{S}_{a b}(k, 0)$ in Eq. (13). This is the reason for using the same notation as in Eq. (14). In the sequel it is convenient to also use the notation,

$$
\mathbf{G}^{+}(\mathbf{r}, t)=\mathbf{G}(\mathbf{r}, t)-\mathbf{S}^{\infty}(t) \delta(\mathbf{r}),
$$

where $G^{+}$represents the correlation function on scales larger than a diameter. The structure factors $\widetilde{\mathbf{S}}^{+}(\mathbf{k}, \tau)$ in Eq. (19) can be Fourier inverted. However, the functions $\widetilde{\mathbf{S}}(\mathbf{k}, \tau)$ in Eq. (14) contain a part $\widetilde{\mathbf{S}}(\mathbf{k}, 0)$, which is independent of $k$ in the relevant $k$ interval, and which yields after Fourier inversion a contribution proportional to $\delta(\mathbf{r})$. These "large- $k$ ", contributions are in fact the correlation functions of an elastic hard sphere (EHS) fluid as $k \rightarrow 0$, i.e.,

$$
\begin{gathered}
G_{n n}(\mathbf{r}, t) \simeq \frac{n}{2 c_{T}^{2}} \delta(\mathbf{r}), \\
G_{T T}(\mathbf{r}, t) \simeq \frac{2 T^{2}(t)}{d n} \delta(\mathbf{r}), \\
G_{\alpha \beta}(\mathbf{r}, t) \simeq \frac{T(t)}{m n} \delta(\mathbf{r}) \delta_{\alpha \beta} .
\end{gathered}
$$

According to Sec. II, "large $k$ " means here $\sqrt{\gamma_{0}} / l_{0}<k$ $<\min \left\{2 \pi / l_{0}, 2 \pi / \sigma\right\}$. Here $G_{n n}$ is the coarse-grained densitydensity correlation function for EHS, in which the Fourier components with $k \sigma \gtrsim 2 \pi$ have been discarded. In Appendix $\mathrm{B}$ we derive the formulas, necessary for the analytic and numerical Fourier inversion of $\mathbf{S}^{+}(\mathbf{k}, t)$, as defined in Eq. (26). After these preparations we analyze the numerical solutions.

\section{Numerical solutions}

The numerical evaluation of structure factors in Eqs. (12) and (22) with or without Langevin noise have been performed using MATHEMATICA. The values of $S_{a b}(\mathbf{k}, t)$ "with noise" are plotted as solid lines in Figs. 5, 6, and 7 for different components $(a b)$; those "without noise" as dashed lines.

The qualitative features of the noisy and noiseless theory (solid and dashed lines in the figures) are about the same for small- $k$ values $\left(k l_{0}<\gamma_{0}\right)$ as shown for $S_{n n}$ in Fig. 5(a) and for $S_{\|}=S_{l l}$ in Fig. 6(a). However, the predictions differ substantially at large- $k$ values, where the results of the noiseless theory do not approach the plateau values $\mathbf{S}(\mathbf{k}, 0)$, but vanish. The reason for this incorrect prediction has been explained above (22).

For the transverse structure factor $S_{\perp}$ [upper solid and dashed lines in Fig. 6(b)] the noisy and noiseless theory are quantitatively different except in the limit $k \rightarrow 0$. In general, in the long-wavelength and large time limit the results of both theories approach each other. 

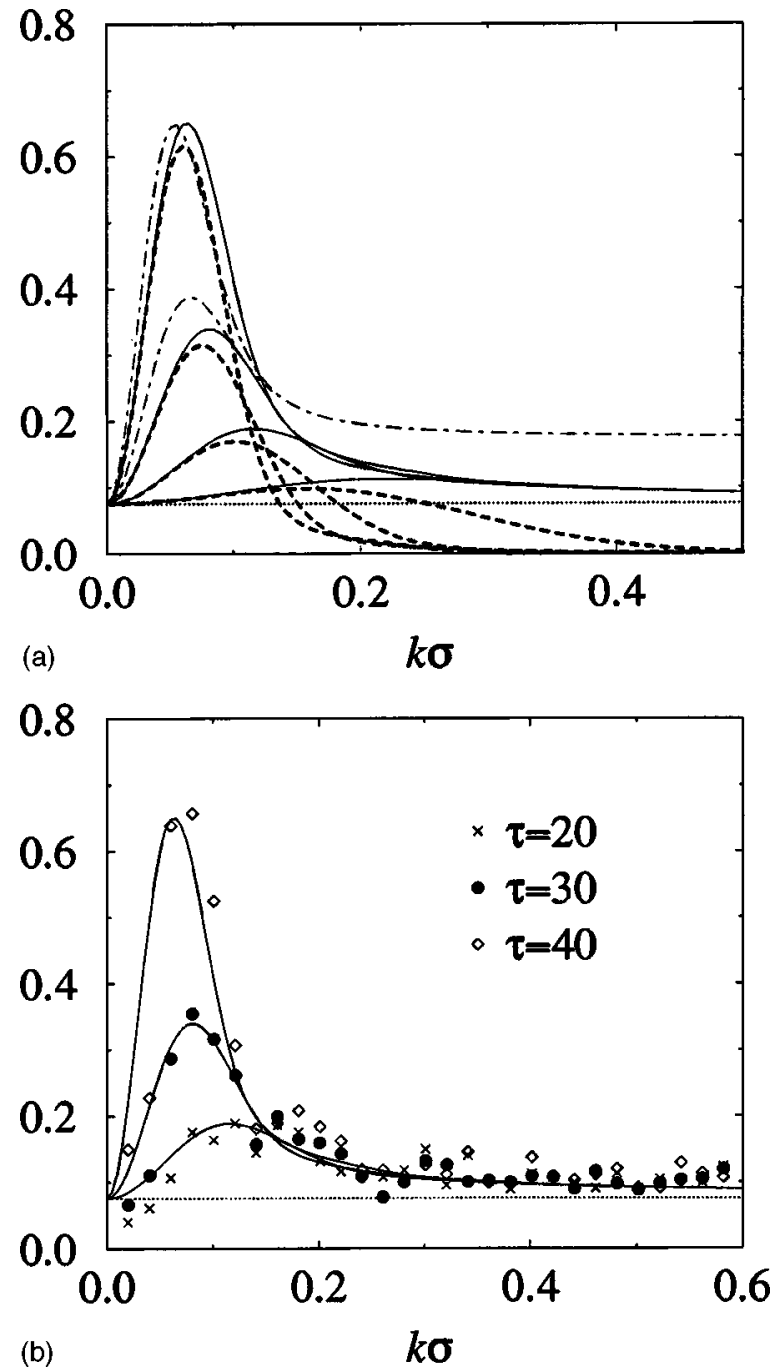

FIG. 5. Density structure factor $S_{n n}$, in units $1 / \sigma^{2}$, versus $k \sigma$ for $\phi=0.4$ and $\alpha=0.9$, at $\tau=10,20,30$, and 40 collisions per particle, exhibits the clustering instability with a growing maximum at $k_{\max }(t)$, which shifts to the left when $\tau$ increases. Solid and dashed lines are the numerical solutions of Eqs. (12) and (22) with and without Langevin noise, respectively. They differ appreciably, except at small $k$. The simple analytic approximation (37), shown in (a) as dashed-dotted lines for $\tau=30$ and 40, gives a good description in the long-time and long-wavelength limit. (b) Numerical solution of Eq. (12) compared with MD simulation results (courtesy of J.A.G. Orza et al. [4]).

To understand the totally different behavior of density and velocity fluctuations, we consider the solid lines in Figs. 5(a) and 6(a), 6(b) for $S_{n n}(k, t)=n^{2} \widetilde{S}_{n n}(k, t)$ and $S_{a}(k, t)$ $=\mathrm{v}_{0}^{2}(t) \widetilde{S}_{a}(k, t) \quad$ with $\quad a=(\perp, \|)$, where $\frac{1}{2} m \mathrm{v}_{0}^{2}(t)=T(t)$ $=T_{0} \exp \left[-2 \gamma_{0} \tau\right]$. Their square roots give the actual size of the fluctuations in the density and flow fields. The density fluctuations for large $k$ remain at the constant thermal noise level. However, at small wave vectors the density fluctuations are unstable and increase in size. The maximum in $S_{n n}$ at $k_{\max }(t)=2 \pi / L_{c l}(t)$ sharpens up and shifts to smaller wave vectors, where $L_{c l}(t) \sim \sqrt{\tau}$ for the full range of $\tau$ values, as determined numerically. So, the present theory predicts a growing length scale $L_{c l}(t)$ of density clusters in undriven IHS fluids in the early stages of cluster formation.
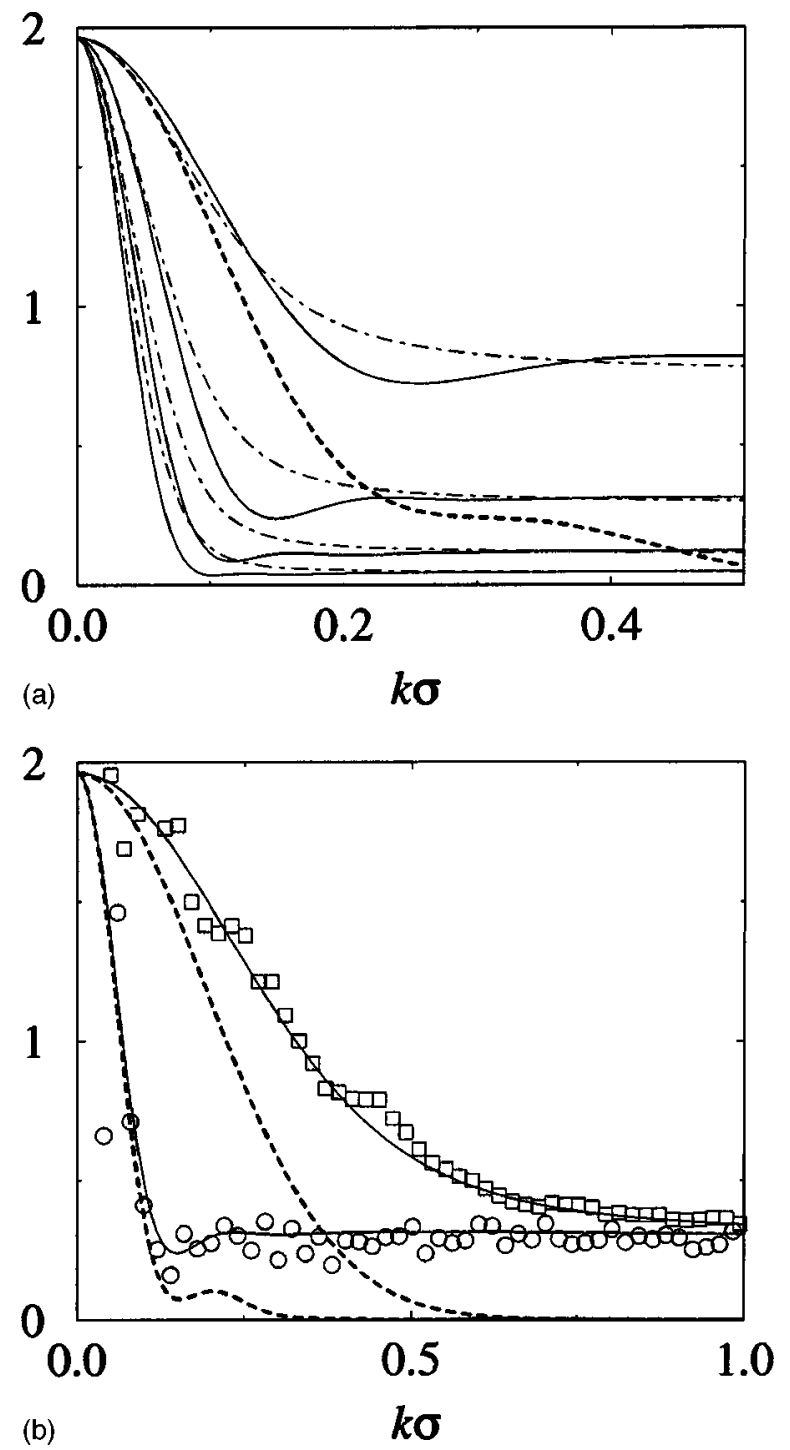

FIG. 6. Structure factors of velocity fluctuations $S_{\|}$and $S_{\perp}$, in units $T_{0} \sigma^{2} / m$, versus $k \sigma$ for $\phi=0.4$ and $\alpha=0.9$ illustrate the phenomenon of noise reduction at small wavelengths. The initial value $S_{\|}(k, 0)=S_{\perp}(k, 0)=T_{0} / \rho$ is a horizontal line, tangent to the maximum at $k=0$. Top panel (a) plots $S_{\|}$at $\tau=10,20,30$ and 40 (lines labeled from top to bottom), where solid lines represent the numerical solution of Eq. (12), and the dashed-dotted lines represent the approximate analytic result (31), which is only valid for $\tau>1 / \gamma_{0}$ $\simeq 21$. The dashed line represents the numerical solution of the "noiseless" Cahn-Hilliard theory (22) at $\tau=10$, which deviates substantially from the solid line at $\tau=10$, except near $k=0$. (b) Comparison with MD simulation results (courtesy of J.A.G. Orza et al. [4]) at $\tau=20$ for $S_{\perp}$ (squares) and $S_{\|}$(circles).

We return to the density instability in Sec. V A.

Next we consider the velocity fluctuations $S_{a}(k, t)$ with $a=(\perp, \|)$, which are for all $\tau, k$ smaller than the initial value $S_{a}(k, 0)=T_{0} / \rho$ [see Figs. 6(a) and 6(b)]. Consequently, the fluctuations in the flow field do not grow, but are at all times stable and bounded by the noise level in the initial equilibrium state. The plateau value $T(t) / \rho$ at fixed large- $k$ values decreases in time with the cooling temperature. In Sec. IV A we return to the mechanism responsible for the growing vortices, shown in Fig. 1(a). It is also of interest to observe that the locations of the maxima of $S_{n n}$ in Fig. 5 and those of the 

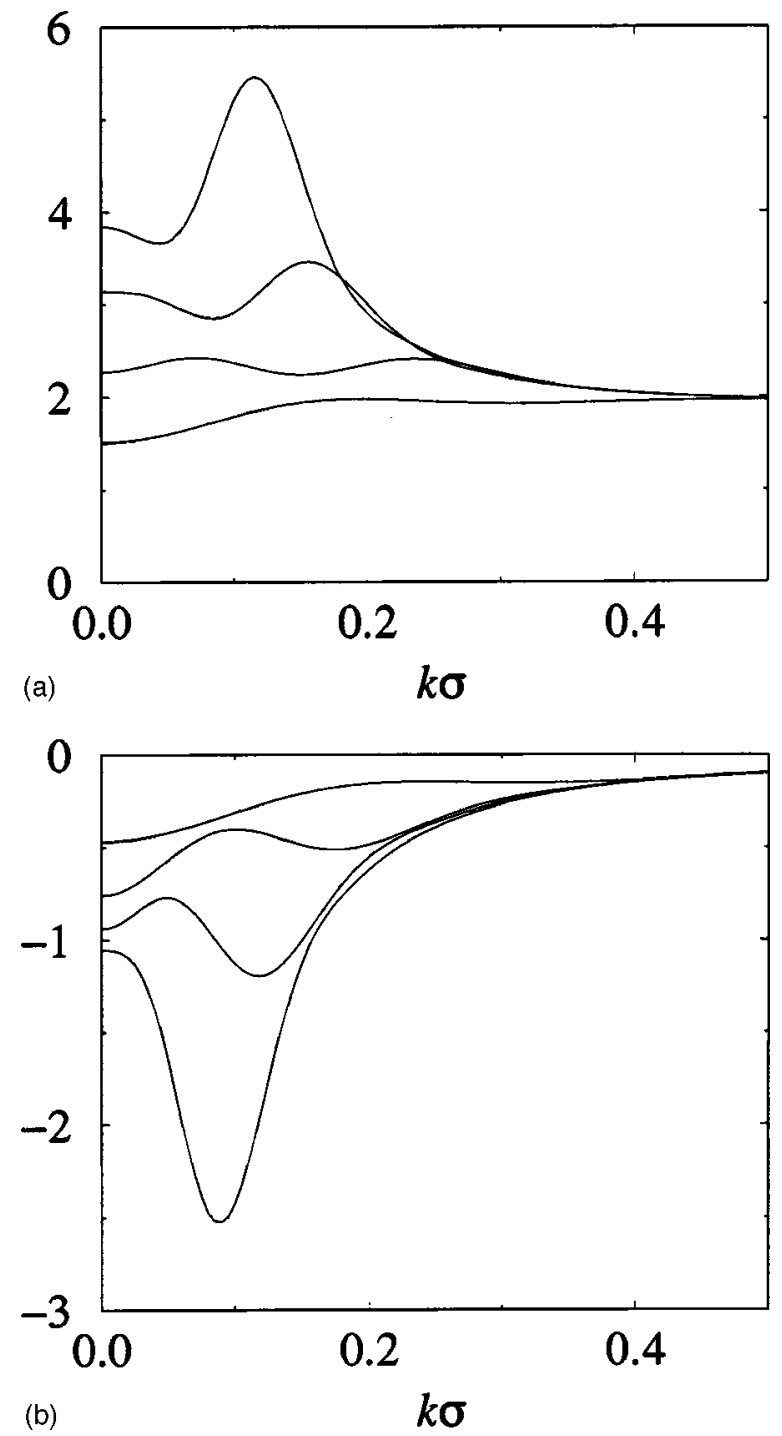

FIG. 7. Rescaled structure factors (a) $\widetilde{S}_{T T}$ and (b) $\widetilde{S}_{n T}$, all in units $\sigma^{2}$, versus $k \sigma$ for same the parameters as used in Fig. 5. The structure factor in (b) vanishes initially and develops structure as time increases. $\widetilde{S}_{T T}$ develops structure on top of its initial (plateau) value $S_{T T}(k, 0)=2 / d n \simeq 1.96$.

minima ("dip") of $S_{\|}$in Fig. 6 approximately coincide. It indicates that the density instability is closely connected to the dynamics controlling $S_{\|}$, which turns out to be the heat mode, as we will show in the next section.

The numerical values of rescaled temperature fluctuations $\widetilde{S}_{T T}$ and crosscorrelation $\widetilde{S}_{n T}$ are shown in Figs. 7(a) and $7\left(\right.$ b). We first observe that $\widetilde{S}_{T T}$ and $\widetilde{S}_{n T}$ show roughly the same behavior as $\widetilde{S}_{n n}$ (except at small $k$ ) with a maximum, that shifts towards smaller $k$ with increasing time. This suggests that the dynamics for $(n n),(T T)$, and $(n T)$ fluctuations is controlled by the same mode, as will be shown in Sec. V A. An interesting feature, shown by $\widetilde{S}_{n T}$, is that density and temperature fluctuations in granular fluids are anticorrelated at all wavelengths. This property was also noted by McNamara [12] in his analysis of the macroscopic hydrodynamic modes of granular fluids, and is intuitively clear. When a random fluctuation creates locally an excess density, the collision rate, $\omega \propto n$, increases locally, which enhances the collisional dissipation and lowers the temperature. This mechanism is in fact at the basis of the "phase separation" of the homogeneous granular fluid into cold dense clusters surrounded by a hot dilute granular gas. We have also calculated the nonvanishing crosscorrelations $\widetilde{S}_{n l}$ and $\widetilde{S}_{T l}$, which are purely imaginary for symmetry reasons.

An illustration of the comparison with the MD simulations on systems of 50000 inelastic hard disks of Refs. $[1,2,4]$ is shown in Fig. 5(b) for $S_{n n}$ and in Fig. 6(b) for $S_{\perp}$ and $S_{\|}$. The agreement between theory and simulations is in general very good, even for rather large inelasticities ( $\alpha$ $\simeq 0.6$ ). By comparing the simulation results for $S_{\perp}$ and $S_{\|}$in Fig. 6(b) with the numerical results of the theory with noise (solid lines) and without (dashed lines), we have observed that the agreement in the "noisy" case extends over the full range of $k$ values, whereas in the "noiseless" case it is restricted to the small- $k$ range, which is indeed very small for the transverse structure factor $S_{\perp}(k, t)$. Similar conclusions hold when comparing the simulation results for $S_{n n}(k, t)$ in Fig. 5(b) with the numerical results in Fig. 5(a) with and without Langevin noise included. Consequently the noiseless Cahn-Hilliard theory does not agree quantitatively with the simulation results.

So far we have established by a rather complex numerical procedure that the Langevin equations for granular fluids give predictions that agree quantitatively with MD simulations. Our next goal is to understand theoretically which excitations are responsible for the observed behavior. This will be done in Secs. IV and V.

\section{FLOW FIELD PROPERTIES}

\section{A. Transverse and longitudinal structure factors}

We first consider the simplest case of the transverse structure factor $S_{\perp}(k, t)=\mathrm{v}_{0}^{2}(t) \widetilde{S}_{\perp}(k, \tau)$ with $(a b)=(\perp \perp)$. It describes the transverse velocity or vorticity fluctuations $\tilde{u}_{\perp}(\mathbf{k}, \tau)$, which are decoupled from the remaining Fourier modes, and satisfy a one-component Langevin equation, where the matrix $\mathbf{M}(\mathbf{k})$ in Eq. (8) reduces to a single number $\zeta_{\perp}(k)=\gamma_{0}\left(1-\xi_{\perp}^{2} k^{2}\right)$. The complete structure factor is readily found from Eqs. (20), (14), and (15), and yields [1]

$$
S_{\perp}(k, t)=\frac{T(t)}{\rho}\left(1+\frac{\exp \left[2 \gamma_{0}\left(1-\xi_{\perp}^{2} k^{2}\right) \tau\right]-1}{1-\xi_{\perp}^{2} k^{2}}\right) .
$$

This expression is plotted as the upper solid line in Fig. 6(b). It does not grow, but slowly decays at large $\tau$ as $S_{\perp}(k, t)$ $\simeq\left(T_{0} / \rho\right) \exp \left(-2 \gamma_{0} \xi_{\perp}^{2} k^{2} \tau\right) /\left(1-\xi_{\perp}^{2} k^{2}\right)$, the faster the larger $k$. At the largest wavelengths $\left(\xi_{\perp}^{2} k^{2} \ll 1\right)$ it simply represents vorticity diffusion on the "internal" time scale $\tau$, with a diffusivity $\gamma_{0} \xi_{\perp}^{2}=\nu / \omega$. Therefore, the typical length scale of vortices grows like $L_{\mathrm{v}}(t) \sim 2 \pi \xi_{\perp} \sqrt{2 \gamma_{0} \tau} \sim 2 \pi \sqrt{\nu \tau / \omega}$, which is independent of the degree of inelasticity.

Next, we consider the rescaled longitudinal structure factor $\widetilde{S}_{\|}(k, t)$ with $(a b)=(l l)$, where the most dominant term for large $\tau$ in $(20)$ is $(\lambda \mu)=(H H)$, as can be seen from Fig. 3 . There is no coupling to the shear modes. This leads in the structure factor $S_{\|}(k, t)=\mathrm{v}_{0}^{2}(t) \widetilde{S}_{\|}(k, \tau)$ to a slowly decaying contribution with an overall decay rate proportional to $k^{2}$. 
All remaining contributions decay faster; at least as fast as $\mathrm{V}_{0}(t) \sim \exp \left(-\gamma_{0} \tau\right)$, provided $\tau \gg 1 / \gamma_{0}$. The slowest decay occurs at very long wavelengths, i.e., in the dissipative range $\left(k l_{0} \ll \gamma_{0}\right)$. To calculate the dominant $(H H)$ contribution to Eq. (20) we need the coefficient $\mathcal{B}_{l l}^{H H}(k)$. It follows for small $k$ from Eqs. (21), (18), and (A8), and is given by

$$
\mathcal{B}_{l l}^{H H}(k)=2 \gamma_{0} \widetilde{S}_{l l}(k, 0)=\frac{\gamma_{0}}{n} .
$$

Inserting these data in Eq. (20) and combining it with Eq. (14) yields for $k l_{0} \ll \gamma_{0}$ and $\gamma_{0} \tau \gg 1$,

$$
S_{\|}(k, t)=\frac{T(t)}{m n}\left(1+\frac{\exp \left[2 \gamma_{0}\left(1-\xi_{\|}^{2} k^{2}\right) \tau\right]-1}{1-\xi_{\|}^{2} k^{2}}\right),
$$

where the dispersion relation $\zeta_{H}(k)=\gamma_{0}\left(1-\xi_{\|}^{2} k^{2}\right)$ has been used. Note that the analytic $(H H)$ approximation is only valid for $\gamma_{0} \tau \gg 1$, whereas the numerical result is valid for all $\gamma_{0} \tau$. This long-wavelength and long-time approximation for $S_{\|}$has the same form as the exact result (29) with $\xi_{\perp}$ replaced by $\xi_{\|}$. In Fig. 6(a) we compare the result (31) (dotdashed lines) with the numerical solution (solid lines), presented in Sec. III A. It is straightforward to also obtain an analytic approximation, which applies in the elastic $k$ range, where $k l_{0} \propto \sqrt{\gamma_{0}}$. However, we do not show this result as the extrapolation of the simple small- $k$ approximation (31) captures for $\gamma_{0} \tau>1$ the global features at small $k$ as well as the plateau values at larger- $k$ quantitatively. It misses, however, the little dip at intermediate- $k$ values (see, however, Sec. V).

The behavior of the longitudinal structure factor on the largest length scales and for times $\tau \gg 1 / \gamma_{0}$ follows again from Eq. (31) as $S_{\|}(k, t) \simeq\left(T_{0} / \rho\right) \exp \left(-2 \gamma_{0} \xi_{\|}^{2} k^{2} \tau\right)$. This implies that the heat mode on the largest spatial and temporal scales is a purely diffusive mode with a diffusivity $\gamma_{0} \xi_{\|}^{2}$. It is much larger than the diffusivity $\gamma_{0} \xi_{\perp}^{2}$ for the vorticity (see Fig. 4), and the associated length scale grows like $L_{\|}(t) \sim 2 \pi \xi_{\|} \sqrt{2 \gamma_{0} \tau}$. Inspection of the eigenmodes $\left\{\tilde{\mathbf{w}}_{H}(\mathbf{k}), \tilde{\mathbf{v}}_{H}(\mathbf{k})\right\}$ in Eqs. (A8) of Appendix A for $k \rightarrow 0$ shows that this diffusive mode is a purely longitudinal velocity field $\tilde{u}_{l}(\mathbf{k}, \tau)$. Its diffusivity $\gamma_{0} \xi_{\|}^{2}$, defined in Eq. (A4), depends for small inelasticities $\left(\gamma_{0} \rightarrow 0\right)$ mainly on thermodynamic variables, like compressibility and pressure, and only slightly on transport coefficients.

The physical implications of Fig. 6 are quite interesting. It shows the phenomenon of noise reduction [17] at small wavelengths. With increasing time the fluctuations $S_{\|}(k, t)$ and $S_{\perp}(k, t)$ in the flow field decrease at larger- $k$ values and remain for all $k$ bounded by their initial equipartition value $T_{0} / \rho$, which is independent of $k$. This can be rephrased by stating that the flow field exhibits only a "relative" instability. The noise reduction is a direct consequence of the microscopic inelastic collision dynamics, which forces the particles to align more and more in successive collisions. It is this "physical coarse graining" process that selectively suppresses the shorter-wavelength fluctuations in the flow field in an ever-increasing range of wavelengths. Consistent with this picture is also the selective suppression of the divergence of the flow field $u_{\|}(\mathbf{k}, t)$, which decays at a much faster rate $\gamma_{0} \xi_{\|}^{2} k^{2}$ than its rotational part $u_{\perp}(\mathbf{k}, t)$ that decays with a rate $\gamma_{0} \xi_{\perp}^{2} k^{2}$. So, noise reduction is the pattern selection mechanism, responsible for the growing vortex structures observed in Fig. 1. The mechanism for pattern formation that creates the peaks in $S_{\perp}(k, t)$ and $S_{\|}(k, t)$ at $\mathbf{k}=0$ in reciprocal space is, in fact, very similar to the process of peneplanation in structural geology [6] for peak formation in real space, where the earth surface around the peak is gradually being removed by selective erosion. The formation of Mount Uluru (Ayers Rock) in the center of Australia is an outstanding example of peneplanation.

\section{B. Correlations in incompressible flows}

There is an interesting limiting case of the theory, the incompressible limit [1], that greatly simplifies and elucidates the analytic solution of the full set of coupled linearized equations (8) for hydrodynamic fluctuations. It is well known from fluid dynamics and the theory of turbulence $[33,20]$ that ordinary elastic fluid flows are quite incompressible. This implies that $\boldsymbol{\nabla} \cdot \mathbf{u}=0$ and as a consequence the longitudinal mode $u_{l}(\mathbf{k}, t) \simeq 0$. Then, the nonlinear Eq. (1) for the transverse flow field or, equivalently, for the vorticity, practically decouples from the remaining hydrodynamic equations. In the comoving reference frame there is only a nonlinear coupling of the temperature fluctuations to the transverse flow field through the nonlinear viscous heating, $\eta|\nabla \mathbf{u}|^{2}$. We therefore expect that the IHS fluid in the nearly elastic case can be considered as incompressible, at least to lowest approximation.

What are the consequences of this assumption? The structure factor $S_{n n}(k, t)$ of density fluctuations does not evolve in time on account of Eqs. (A1) and (A2). The temperature fluctuation $\delta T(\mathbf{k}, t)$ in Eq. (A1) simply decays as a kinetic mode and the average temperature stays spatially homogeneous. Clearly, the assumption is too drastic a simplification to describe the density and temperature fluctuations. However, an approximate theory based on vorticity fluctuations alone is justified to describe the correlations in the flow field, as discussed in Sec. IV A.

So, we combine the assumption of incompressibility, $S_{\|}(k, t)=0$, with $S_{\perp}(k, t)$ in Eq. (29), using Eqs. (24) and (25). This enables us, for thermodynamically large systems, to explicitly calculate the correlation functions $G_{\alpha \beta}(\mathbf{r}, t)$ of the velocity field, by inverse Fourier transformation, i.e.,

$$
\begin{aligned}
G_{\alpha \beta}^{+}(\mathbf{r}, t) & =\hat{r}_{\alpha} \hat{r}_{\beta} G_{\|}^{+}(r, t)+\left(\delta_{\alpha \beta}-\hat{r}_{\alpha} \hat{r}_{\beta}\right) G_{\perp}^{+}(r, t) \\
& =\int \frac{d \mathbf{k}}{(2 \pi)^{d}} \exp (i \mathbf{k} \cdot \mathbf{r})\left(\delta_{\alpha \beta}-\hat{k}_{\alpha} \hat{k}_{\beta}\right) S_{\perp}^{+}(k, t) .
\end{aligned}
$$

The Fourier transform has been calculated in Appendix B, and yields for the two scalar functions $G_{\lambda}^{+}(r, t)$ with $\lambda$ $=\{\|, \perp\}$

$$
G_{\lambda}^{+}(r, t)=\frac{T(t)}{m n \xi_{\perp}^{d}} g_{\lambda}\left(\frac{r}{\xi_{\perp}}, 2 \gamma_{0} \tau\right)
$$

where $g_{\lambda}(x, s)$ is given in Eqs. (B11) of Appendix B. Both functions are not independent, but $g_{\perp}$ can be calculated from 


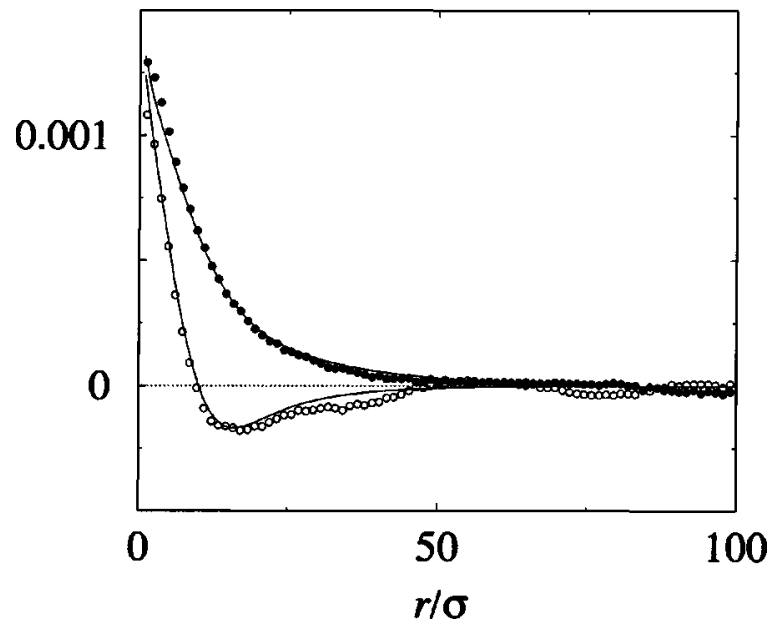

FIG. 8. Comparison of theoretical predictions (34), based on incompressibility, with MD simulation results for $G_{\|}$(filled circles) and $G_{\perp}$ (open circles), in units $T_{0} / m$, versus $r / \sigma$. Parameter values are $\phi=0.4, \alpha=0.9$, and $\tau=40$.

$g_{\|}$using Eq. (B7). This is a well-known relation in the theory of homogeneous and isotropic turbulence in incompressible flows (see Refs. [33] and [19], Chap. 3).

As an explicit example we show the result for inelastic hard disks in Fig. 8, which is most relevant for a comparison with existing computer simulations. Their analytic form is

$$
g_{\|}(x, s)=\frac{1}{2 \pi x^{2}} \int_{0}^{s} d s^{\prime} e^{s^{\prime}-x^{2} / 4 s^{\prime}},
$$

and $g_{\perp}=\partial\left(r g_{\|}\right) / \partial r$. The function $g_{\perp}(x, s)$ has a negative minimum, while $g_{\|}(x, s)$ is positive for all $x, s, d$; there are algebraic tails $g_{\|}(x, s) \sim-(d-1) g_{\perp}(x, s) \sim C x^{-d}$ with a coefficient $C$ and a correction term of $\mathcal{O}\left[\exp \left(-x^{2} / 4 s\right)\right]$, explicitly given in Eq. (B12). These functions have structure on hydrodynamic space and time scales where both $x=r / \xi_{\perp}$ and $s=2 \gamma_{0} \tau$ can be either large or small with respect to unity. At small inelasticity $\left(\gamma_{0} \rightarrow 0\right)$ the dynamic correlation length $\xi_{\perp}$ and mean free path $l_{0}$ are well separated.

Long-range spatial correlations in systems with shortrange interactions are a generic feature of nonequilibrium steady states (NESS) (see the reviews on driven diffusive systems [8] or systems with imposed temperature gradients [34]). Two remarks are in order here. Firstly, the presence of long-range spatial correlations, as found in granular fluids, shows that these typical results for NESS carry over to classes of adiabatically changing states, such as here the homogeneous cooling state where the temperature changes adiabatically. Secondly, an essential feature for the existence of long spatial tails in NESS is a breaking of the isotropic spatial symmetry [34]. In incompressible flows this symmetry is broken by setting $u_{l}(\mathbf{k}, t)=0$, and keeping only the transverse velocity field.

A more systematic comparison between the theoretical predictions (34) and molecular dynamics simulations is made in Refs. [1,4]. In Fig. 8 we show the results from a single simulation run at packing fraction $\phi=0.4$, and small inelasticity $\alpha=0.9$. The parallel part $G_{\|}(r, t)$ exhibits a tail $\sim r^{-d}$ [see Fig. 2(a) in Ref. [1]] and shows good agreement up to $\tau=100$, which is well beyond the crossover time $\tau_{c}=67$ (de- fined in Fig. 2) that separates the linear regime from the nonlinear clustering regime. The minimum in $G_{\perp}(r, t)$ at $L_{\mathrm{v}}(t)$ can be identified as the mean diameter of vortices, shown in Fig. 1. The analytic result for $G_{\perp}(r, t)$ in Eq. (34) for large times shows that that $L_{\mathrm{V}}(t) \sim 2 \pi \xi_{\perp} \sqrt{2 \gamma_{0} \tau}$ is growing through vorticity diffusion.

Apart from the restrictions to hydrodynamic space and time scales, there are two essential criteria limiting the validity of the incompressible theory: (i) system sizes $L$ must be thermodynamically large $\left(L \gg 2 \pi \xi_{\perp}\right)$, so that Fourier sums over $\mathbf{k}$ space can be replaced by $\mathbf{k}$ integrals. (ii) In principle, times must be restricted to the linear hydrodynamic regime $\left(\tau \lesssim \tau_{c}\right)$, so that the system remains close to the HCS. In fact, the simulations show that our description of the fluctuations in terms of a Langevin equation based on incompressibility is correct over a time range much larger than $\tau_{c}$. This is understandable because velocity correlations do not grow in amplitude (peneplanation), and are only weakly coupled to the exponentially growing density fluctuations.

\section{Correlations in compressible flows}

In this section we extend the theory to compressible flows [2]. The description of the velocity fluctuations $G_{\alpha \beta}(\mathbf{r}, t)$ in Sec. IV B was based on fluctuating hydrodynamics for the vorticity fluctuations only, i.e., the absence of longitudinal fluctuations (incompressibility assumption). Figure 6(b) confirms that this assumption is very reasonable indeed for nearly elastic fluids, as $S_{\|}^{+}(k, t)=S_{\|}(k, t)-T / \rho$ is vanishingly small down to very small- $k$ values. However, for the smallest wave numbers, the incompressibility assumption breaks down. As the analysis of Eqs. (29) and (31), as well as the numerical evaluation in Fig. 6 show, the structure factors $S_{\|}(k, t)$ and $S_{\perp}(k, t)$ become equal as $k \rightarrow 0$. This implies for large distances $G_{\alpha \beta}(\mathbf{r}, t) \sim S_{\perp}(k \rightarrow 0, t) \delta_{\alpha \beta} \delta(\mathbf{r})$, and thus the absence of algebraic long-range correlations on the largest scales $\left(r \gg 2 \pi \xi_{\|}\right)$. Therefore, we can already conclude that the asymptotic behavior of $G_{\perp}(r, t)$ and $G_{\|}(r, t)$ cannot be $r^{-d}$. Instead the $r^{-d}$ tail, obtained in Sec. IV B, describes intermediate behavior which is exponentially cut off at a distance determined by the width of $S_{\|}^{+}(k, t)$. This width can be estimated from the eigenvalues of the hydrodynamic matrix, more precisely from the dispersion relation of the heat mode, which is a purely longitudinal velocity $\tilde{u}_{l}$ for $k \rightarrow 0$. To second order in $k$ its dispersion relation is given by $\zeta_{H}(k)=\gamma_{0}\left(1-k^{2} \xi_{\|}^{2}\right)$. Note that for small inelasticities $\xi_{\|}$and $\xi_{\perp}$ are well separated (see Fig. 4), as $\xi_{\|} \sim 1 / \gamma_{0}$, whereas $\xi_{\perp}$ $\sim \xi_{l} \sim \xi_{T} \sim 1 / \sqrt{\gamma_{0}}$.

Using the analytic approximation (31) for $S_{\|}(k, t)$, valid for small $k$ and large $\tau$, the structure factor $S_{\alpha \beta}^{+}(\mathbf{k}, t)$ can be written as

$$
\begin{aligned}
S_{\alpha \beta}^{+}(\mathbf{k}, t) \approx & \frac{T(t)}{\rho} \int_{0}^{s} d s^{\prime} e^{s^{\prime}}\left[\hat{k}_{\alpha} \hat{k}_{\beta} \exp \left(-s^{\prime} k^{2} \xi_{\|}^{2}\right)\right. \\
& \left.+\left(\delta_{\alpha \beta}-\hat{k}_{\alpha} \hat{k}_{\beta}\right) \exp \left(-s^{\prime} k^{2} \xi_{\perp}^{2}\right)\right]
\end{aligned}
$$

where $s=2 \gamma_{0} \tau$. If the system is thermodynamically large $\left(L \gg 2 \pi \xi_{\|}\right), G_{\|}^{+}(r, t)$ and $G_{\perp}^{+}(r, t)$ can be obtained by performing integrals over $\mathbf{k}$ space and yield expressions in terms 

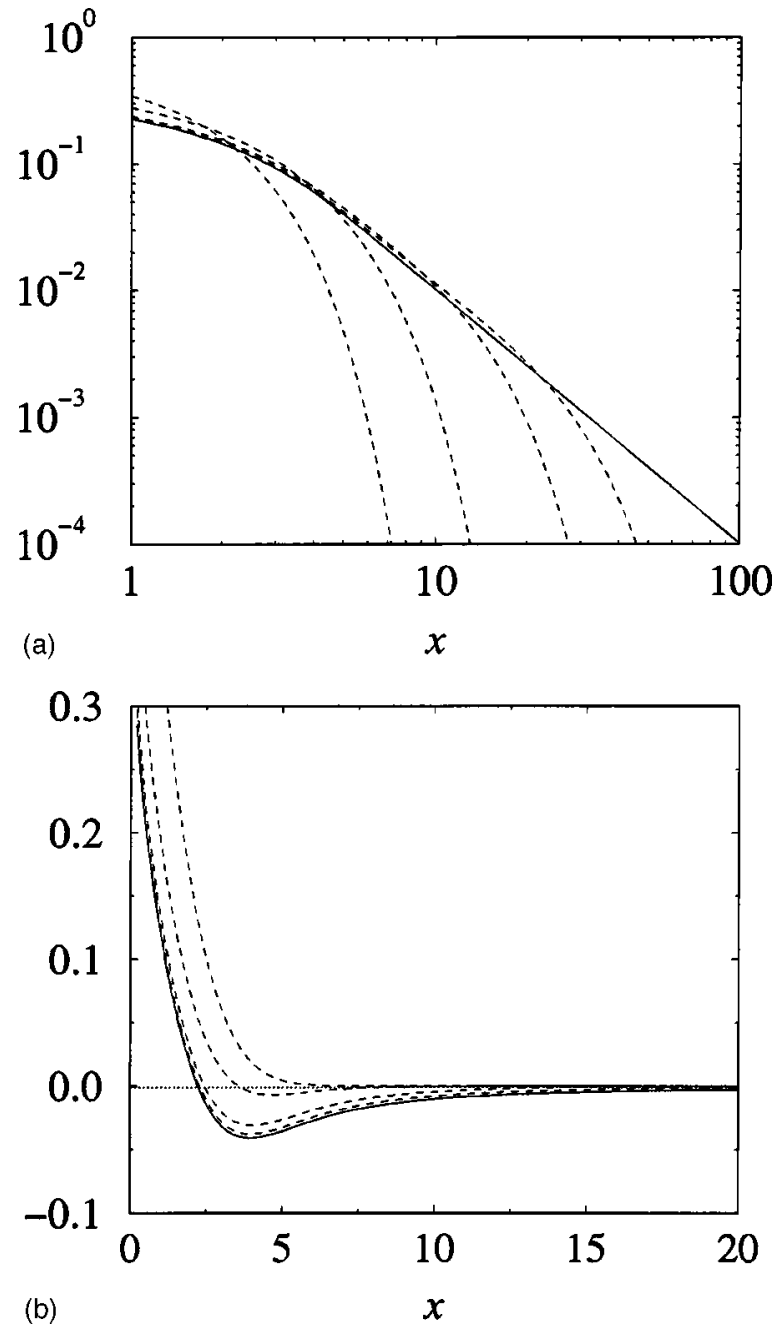

FIG. 9. (a) $g_{\|}(x, s)$ and (b) $g_{\perp}(x, s)$ versus $x=x_{\perp}=r / \xi_{\perp}$ for $s$ $=2 \gamma_{0} \tau=2$. The solid lines correspond to Eq. (34) in the incompressible limit $\left(\xi_{\|} / \xi_{\perp} \rightarrow \infty\right)$, and the dashed lines to approximations (36), for $\xi_{\|} / \xi_{\perp}=1,2,5,10$. As $\xi_{\|} / \xi_{\perp}$ decreases, the $r^{-2}$ tail in (a) is cut off exponentially at smaller distances and finally disappears at $\xi_{\|}=\xi_{\perp}$. The depth of the minimum in (b) decreases with decreasing $\xi_{\|} / \xi_{\perp}$ and finally disappears at $\xi_{\|}=\xi_{\perp}$.

of integrals over simple functions, as derived in Appendix B. Here we only quote the analytic approximation for $d=2$ :

$$
\begin{aligned}
G_{\lambda}^{+}(r, t) \approx & \frac{T(t)}{\rho}\left\{\frac{1}{4 \pi \xi_{\lambda}^{2}} \int_{0}^{s} d s^{\prime} \frac{\exp \left(s^{\prime}-x_{\lambda}^{2} / 4 s^{\prime}\right)}{s^{\prime}}\right. \\
& +\frac{\sigma_{\lambda}}{2 \pi r^{2}} \int_{0}^{s} d s^{\prime} e^{s^{\prime}}\left[\exp \left(-\frac{x_{\|}^{2}}{4 s^{\prime}}\right)\right. \\
& \left.\left.-\exp \left(-\frac{x_{\perp}^{2}}{4 s^{\prime}}\right)\right]\right\}
\end{aligned}
$$

for $\lambda=(\|, \perp)$, where $x_{\lambda}=r / \xi_{\lambda}, \sigma_{\|}=1$, and $\sigma_{\perp}=-1$. We first observe that, in the time regime $\tau \sim 1 / \gamma_{0}, G_{\lambda}^{+}(r, t)$ has structure both on the scale $r \sim 2 \pi \xi_{\perp}$ as well as on $r$ $\sim 2 \pi \xi_{\|}$. Moreover, $G_{\|}^{+}(r, t)$ is positive both in the incompressible as well as in the compressible case because $\xi_{\perp}$ $<\xi_{\|}$. In Fig. 9 we show the above approximations for dif-

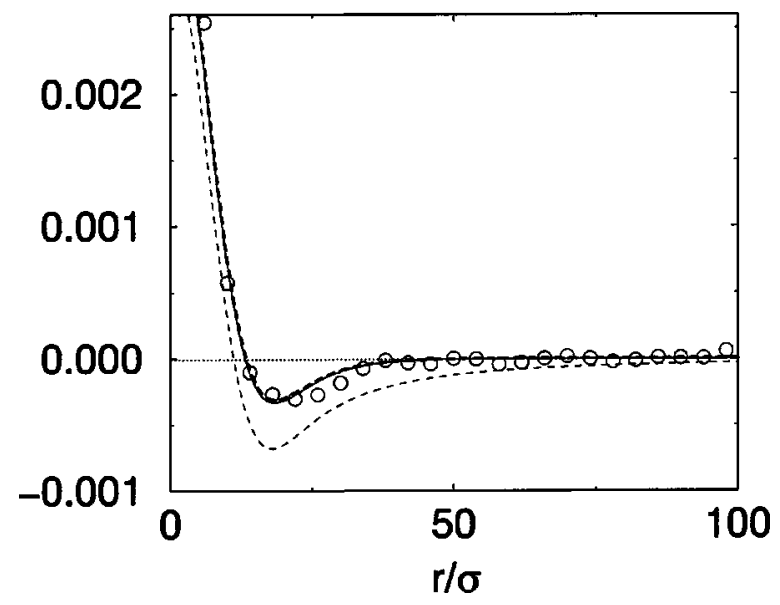

FIG. 10. Perpendicular velocity correlation $G_{\perp}$, in units $T_{0}$ versus $r / \sigma$ for packing fraction $\phi=0.4$, relatively high inelasticity $\alpha$ $=0.6$ and $\tau=40$. Simulation results are compared with prediction (34) of the incompressible theory (dashed line), and the numerical solution (solid line) of the full set of fluctuating hydrodynamic equations.

ferent values of the ratio $\xi_{\|} / \xi_{\perp}$, together with the result $r^{-d}$ for the incompressible limit of Sec. IV B, which is obtained for $\xi_{\|} \rightarrow \infty$. At finite $\gamma_{0}$, Eq. (36) describes exponentially decaying functions at distances $r \gtrsim 2 \pi \xi_{\|}$. Moreover, upon increasing the inelasticity the minimum in $G_{\perp}(r, t)$ becomes less deep and vanishes at $\xi_{\|}=\xi_{\perp}$, and the modifications of the compressible theory become large (see Fig. 10).

The predicted spatial velocity correlations $G_{\|}(r, t)$ and $G_{\perp}(r, t)$ have been obtained by performing inverse Bessel transformations on the numerical results for $S_{\|}(k, t)$ and $S_{\perp}(k, t)$. At small inelasticity ( $\left.\alpha \gtrsim 0.9\right)$ the functions $G_{\|}(r, t)$ and $G_{\perp}(r, t)$, calculated from the full set of hydrodynamic equations, differ for $r \lesssim 2 \pi \xi_{\|}$only slightly from the results for incompressible flow fields (see the discussion in Sec. IV B). However, the algebraic tails $\sim r^{-d}$ in $G_{\|}(r, t)$ and $G_{\perp}(r, t)$ for $r \gtrsim 2 \pi \xi_{\perp}$, as derived in Sec. IV B, are exponentially cut off for $r \gtrsim 2 \pi \xi_{\|}$, as implied by Eq. (36). As the correlation lengths $\xi_{\perp} \sim 1 / \sqrt{\gamma_{0}}$ and $\xi_{\|} \sim 1 / \gamma_{0}$ are well separated for small $\gamma_{0}$, there is an intermediate range of $r$ values where the algebraic tail $\sim r^{-d}$ in $G_{\|}(r, t)$ can be observed.

At higher inelasticity $\xi_{\|}$and $\xi_{\perp}$ are not well separated and, as a consequence, there does not exist a spatial regime in which the longitudinal fluctuations in the flow field can be neglected and the regime of validity of the incompressible theory has shrunk to zero. Figure 10 compares results from incompressible and compressible fluctuating hydrodynamics [the solid line indicates numerical solution; the dashed indicates the for analytic approximation (36)] with simulation data for $G_{\perp}(r, t)$ at $\phi=0.4$ and $\alpha=0.6$, and confirms the necessity of including longitudinal velocity fluctuations to calculate the spatial velocity correlations at reasonably large inelasticities. The agreement between compressible theory and MD simulations is very good, even at large inelasticities.

\section{INSTABILITIES AND DENSITY CLUSTERS}

\section{A. Structure factors}

So far, we have seen that the velocity structure factor, $S_{\perp}(k, t)$ and $S_{\|}(k, t)$, develops a peak by "selective suppres- 
sion" of its large- $k$ components, where the top of the peak remains at its initial value ("peneplanation" in structural geology). Next we will focus on the unstable structure factor $S_{n n}(k, t)$, which describes the density clustering in undriven IHS fluids. A real space analog of this phenomenon of structure formation corresponds to "uplifting" in geological terminology, with the Alps as a typical example. In the comparable case of spinodal decomposition the phase separation is driven by a single unstable mode, the composition fluctuations, described by the macroscopic diffusion equation $\partial_{t} \delta n(\mathbf{k}, t)=z_{D}(k) \delta n(\mathbf{k}, t)$, where the growth rate has the typical form $z_{D}(k)=\Delta k^{2}\left(1-\frac{1}{2} \xi_{D}^{2} k^{2}\right)$. The corresponding structure factor (22) in the noiseless Cahn-Hilliard theory would have the form $S_{n n}(k, t) \sim \exp \left[2 z_{D}(k) t\right]$, and exhibits a maximum growth rate at $k_{\max }=1 / \xi_{D}$, where $z_{D}(k)$ has a maximum. This time-independent length scale fails to describe the growing length scales of the patterns observed in spinodal decomposition [5].

For the granular fluid we first consider a naive version of the noiseless Cahn-Hilliard theory, proposed by Deltour and Barrat [15]. These authors assume that the structure factor $S_{n n}$ can be described by the unstable density field, i.e., $S_{n n}(k, t) \simeq S_{n n}(k, 0) \exp \left[2 \zeta_{H}(k) \tau\right]$, with the growth rate $\zeta_{H}(k)$ of the unstable heat mode. As $\zeta_{H}(k)$ decreases monotonically with $k$, as shown in Fig. 3, this structure factor shows the fastest growth at the smallest wave number $k_{\min }=2 \pi / L$ allowed in a box of length $L$, and does not explain the dynamics of cluster growth.

Next we consider the full theory of Sec. III A with Langevin noise included. The rescaled structure factor $S_{n n}^{+}(k, t)$ in Eq. (20) contains exponentially growing terms, $\exp \left[\left(\zeta_{\lambda}\right.\right.$ $\left.\left.+\zeta_{\mu}\right) \tau\right]$ provided $\zeta_{\lambda}+\zeta_{\mu}>0$. Inspection of the dispersion relations in Fig. 3 shows a fast growth rate $2 \zeta_{H}$ for $k$ below the stability threshold $k_{H}^{*}$, and a much slower growth rate $\left(\zeta_{H}\right.$ $\left.+\zeta_{+}\right)$, less than half of the previous one, that can be neglected for $\gamma_{0} \tau \gg 1$.

So far the arguments are as in Sec. IV C. The big difference is that the size of the fluctuations in the flow field are given by $S_{a}^{+}(k, t)=\mathrm{v}_{0}^{2}(t) \widetilde{S}_{a}^{+}(k, \tau)$ with $a=(\perp, \|)$, where $\mathrm{v}_{0}^{2}(t)$ decreases faster than $\widetilde{S}_{a}^{+}$increases, and there is no growth whatsoever. However, the exponential growth in the density fluctuations $S_{n n}^{+}(k, t)=n^{2} \widetilde{S}_{n n}^{+}(k, \tau)$ is not suppressed by the rescaling factor $n^{2}$. Thus the dominant growth rate in Eq. (20) for $\gamma_{0} \tau \gg 1$ is

$$
S_{n n}(k, t) \simeq \frac{n}{2 c_{T}^{2}}+n^{2} \mathcal{B}_{n n}^{H H}(k) \frac{e^{2 \zeta_{H}(k) \tau}-1}{2 \zeta_{H}(k)},
$$

where $\zeta_{H}$ is given in Eq. (4) and $n / 2 c^{2}$ is the plateau value. We calculate the coefficient $\mathcal{B}_{n n}^{H H}(k)$ in Eq. (21) both for the dissipative and elastic ranges. The eigenmodes $\left\{\tilde{\mathbf{w}}_{H}(\mathbf{k}), \widetilde{\mathbf{w}}_{H}\right.$ $(-\mathbf{k}) t\}$ in Eq. (A8) and the relation $\mathcal{B}_{n n}^{H H}$ $=\left|\tilde{w}_{H n} / \tilde{w}_{H l}\right|^{2} \mathcal{B}_{l l}^{H H}$ in combination with Eq. (30) yield then

$$
\begin{aligned}
\mathcal{B}_{n n}^{H H}(k) & =k^{2} l_{0}^{2} / n \gamma_{0} \quad\left(k l_{0} \ll \gamma_{0}\right) \\
& =\frac{\gamma_{0} d b^{2}}{4 n c_{T}^{2} c_{s}^{4}}\left(\frac{d}{4} a b-c_{T}^{2}\right) \quad\left(k l_{0} \sim \sqrt{\gamma_{0}}\right),
\end{aligned}
$$

with coefficients defined in Eqs. (A3)-(A5).

Equations (37) and (38) for $S_{n n}$ give a fair analytic approximation of the "numerical" solution [solid line in Fig. $5(\mathrm{a})]$ for $\gamma_{0} \tau \gtrsim 2$. The "dissipative" approximation $S_{n n}^{(d i s s)}(k, \tau)$ [dashed-dotted line in Fig. 5(a)] applies for $k \sigma$ $\lesssim 0.12\left(k l_{0} \lesssim 0.9 \gamma_{0}\right)$, and has a maximum for $\gamma_{0} \tau>1$. The "elastic" approximation $S_{n n}^{(e l)}(k, \tau)$ applies for $k \sigma \gtrsim k_{H}^{*} \sigma$ $\simeq 0.16\left(k l_{0} \simeq 1.1 \gamma_{0}\right)$, and its value at $k=k_{H}^{*}$ is for $\tau=40$ $\simeq 1.9 / \gamma_{0}$ and $\tau=80$, respectively, $75 \%$ and $92 \%$ of the corresponding numerical value.

It is instructive to compare the above $k$ values for Fig. 5 (a) with the dispersion relations $\zeta_{H}(k)$ in Fig. 3 for the heat mode which refers to the same density and inelasticity. For wave numbers below the stability threshold $\left(k<k_{H}^{*}\right)$ the density fluctuations grow, and for $k>k_{H}^{*}$ the density fluctuations remain at the thermal noise level. Furthermore, numerical solutions of the theory with [solid lines in Fig. 5(a)] and without noise [dashed lines in Fig. 5(a)] agree in the dissipative range as well, but disagree for $k$ above the threshold $k_{H}^{*}$.

The analytical result (37) demonstrates that the instability is driven through a coupling to the unstable "heat' mode, which is, in the small- $k$ range, a longitudinal velocity mode. The coupling of the density fluctuation to the unstable heat mode is rather weak, $\mathcal{B}_{n n}^{H H} \sim \mathcal{O}\left(k^{2}\right)$, which explains why structure in the flow field appears long before density clusters appear.

The wave number $k_{\max }(t)$ of the maximum growth of $S_{n n}$ in Eq. (37) determines the typical length scale of the density clusters. For $2 \gamma_{0} \tau \gg 1$, it can be determined analytically as $L_{\mathrm{cl}}(t) \sim 2 \pi / k_{\max }(t)=2 \pi \xi_{\|} \sqrt{2 \gamma_{0} \tau}$, which is the same length scale, $L_{\|}(t)$, as appeared in $S_{\|}$. The good agreement between theory and MD simulations, shown in Fig. 5, confirms that the initial growth of density inhomogeneities is indeed controlled by the longitudinal flow field with a length scale $L_{\mathrm{cl}}(t) \sim \sqrt{\tau / \gamma_{0}}$ at small inelasticity $\gamma_{0}$, and not by the transverse flow field with a length scale $L_{\mathrm{v}}(t) \sim 2 \pi \xi_{\perp} \sqrt{2 \gamma_{0} \tau}$ $\sim \sqrt{\tau}$, independent of $\gamma_{0}$.

The pattern selection mechanism for the vortex structures is very different from the mechanism that leads to the formation of density clusters. The latter one is the more common linear instability in density or composition fluctuations, which also occurs in spinodal decomposition [5]; the former one is analogous to peneplanation, as discussed in Sec. IV A.

Finally we consider the temperature fluctuations $\widetilde{S}_{T T}(k, \tau)$ and the crosscorrelation $\widetilde{S}_{n T}(k, \tau)$ in Figs. 7(a) and 7(b). The most dominant contribution to Eq. (20) comes again from two heat modes, and one readily finds that for $\gamma_{0} \tau \gg 1$,

$$
\widetilde{S}_{a b}^{+}(k, \tau)=R_{a b}(k) \widetilde{S}_{n n}^{+}(k, \tau)
$$

with a time-independent coefficient $R(k)$,

$$
R_{n T}(k)=(b-a) / 2 \quad\left(k l_{0} \ll \gamma_{0}\right)=-4 c_{T}^{2} / d b \quad\left(k l_{0} \sim \sqrt{\gamma_{0}}\right),
$$

with coefficients defined in Eq. (A5). Similarly one finds $R_{T T}=R_{n T}^{2}$. This explains qualitatively why the excess structure factors $\widetilde{S}_{T T}^{+}$and $\widetilde{S}_{n T}^{+}$have roughly the same shape as $S_{n n}$. 
On the other hand, the structure factors $\widetilde{S}_{T T}^{+}(0, \tau)$ and $\widetilde{S}_{n T}^{+}(0, \tau)$ at $k=0$ can be calculated directly from Eqs. (9) and (A2), and yield

$$
\begin{gathered}
\widetilde{S}_{T T}(0, \tau)=-\frac{a}{2 n}\left(1-e^{-\gamma_{0} \tau}\right), \\
\widetilde{S}_{n T}(0, t)=\frac{a^{2}}{2 n}\left(1-e^{-\gamma_{0} \tau}\right)^{2}+\frac{2}{d n} e^{-2 \gamma_{0} \tau} .
\end{gathered}
$$

This result explains the $\tau$ variation of both structure factors around $k=0$ as shown in Figs. 7(a) and 7(b). There exist nonuniformities in the $(k, \tau)$ behavior. The limits $k \rightarrow 0$ at fixed $\tau$, and the limit $\tau \rightarrow 0$ at fixed $k$, cannot be interchanged.

\section{B. Spatial correlations}

To obtain predictions for the density-density correlations $G_{n n}(r, t)$, we have to calculate $d$-dimensional Fourier inversions of $S_{n n}(k, t)$, or rather $S_{n n}^{+}(k, t)$, as discussed around Eqs. (26) and (27). As it is a scalar function of $k=|\mathbf{k}|$, its inverse can be reduced to a one-dimensional Bessel transform, as shown in Appendix B in the transition from Eqs. (B2) to (B5).

The Bessel transforms of the numerical data for $S_{n n}(k, t)$, obtained in Sec. III D, have been carried out numerically using MATHEMATICA, and are shown in Fig. 11(a), and similar results for $G_{n T}(r, t)$ are in Fig. 11(b). The spatial density correlation $G_{n n}(r, t)$, obtained numerically from $S_{n n}(k, t)$, exhibits a negative correlation centered around a distance which for large times grows as $\sqrt{\tau}$.

Unlike the case of velocity correlations in Eq. (31), no analytic approximation is available that covers both the small- and large- $k$ behavior of $S_{n n}(k, t)$ correctly. However, the behavior (37) for $k l_{0} \sim \sqrt{\gamma_{0}}\left(\gamma_{0}\right.$ small) and $k l_{0} \ll \gamma_{0}$ is expected to describe correctly the large distance behavior of $G_{n n}(r, t)$ in the range $r \sim 2 \pi l_{0} / \sqrt{\gamma_{0}}$ and $r \gg 2 \pi l_{0} / \gamma_{0}$, respectively. The inverse Fourier transforms of both expressions in Eqs. (37) and (38) can easily be calculated and yield for the dissipative range $\left(r \gg 2 \pi l_{0} / \gamma_{0}\right)$,

$$
\frac{1}{n^{2}} G_{n n}^{>}(r, t) \simeq \frac{l_{0}^{2}}{2 \gamma_{0} n \xi_{\|}^{d+2}} g_{n}^{>}\left(\frac{r}{\xi_{\|}}, 2 \gamma_{0} \tau\right),
$$

and for the elastic range $\left(r \sim 2 \pi l_{0} / \sqrt{\gamma_{0}}\right)$,

$$
\frac{1}{n^{2}} G_{n n}^{<}(r, t) \simeq \frac{\mathcal{B}_{n n}^{H H}}{2 \gamma_{0} \xi_{H}^{d}} g_{n}^{<}\left(\frac{r}{\xi_{H}}, 2 \gamma_{0} \tau\right) \text {. }
$$

All factors on the right-hand side are dimensionless quantities, and the functions $g(x, s)$ are given by

$$
\begin{gathered}
g_{n}^{>}(x, s)=-\frac{e^{s-x^{2} / 4 s}}{(4 \pi s)^{d / 2}}+\int_{0}^{s} d s^{\prime} \frac{e^{s^{\prime}-x^{2} / 4 s^{\prime}}}{\left(4 \pi s^{\prime}\right)^{d / 2}} \\
g_{n}^{<}(x, s)=\int_{0}^{s} d s^{\prime} e^{\zeta_{0} s^{\prime}-x^{2} / 4 s^{\prime}} /\left(4 \pi s^{\prime}\right)^{d / 2}
\end{gathered}
$$
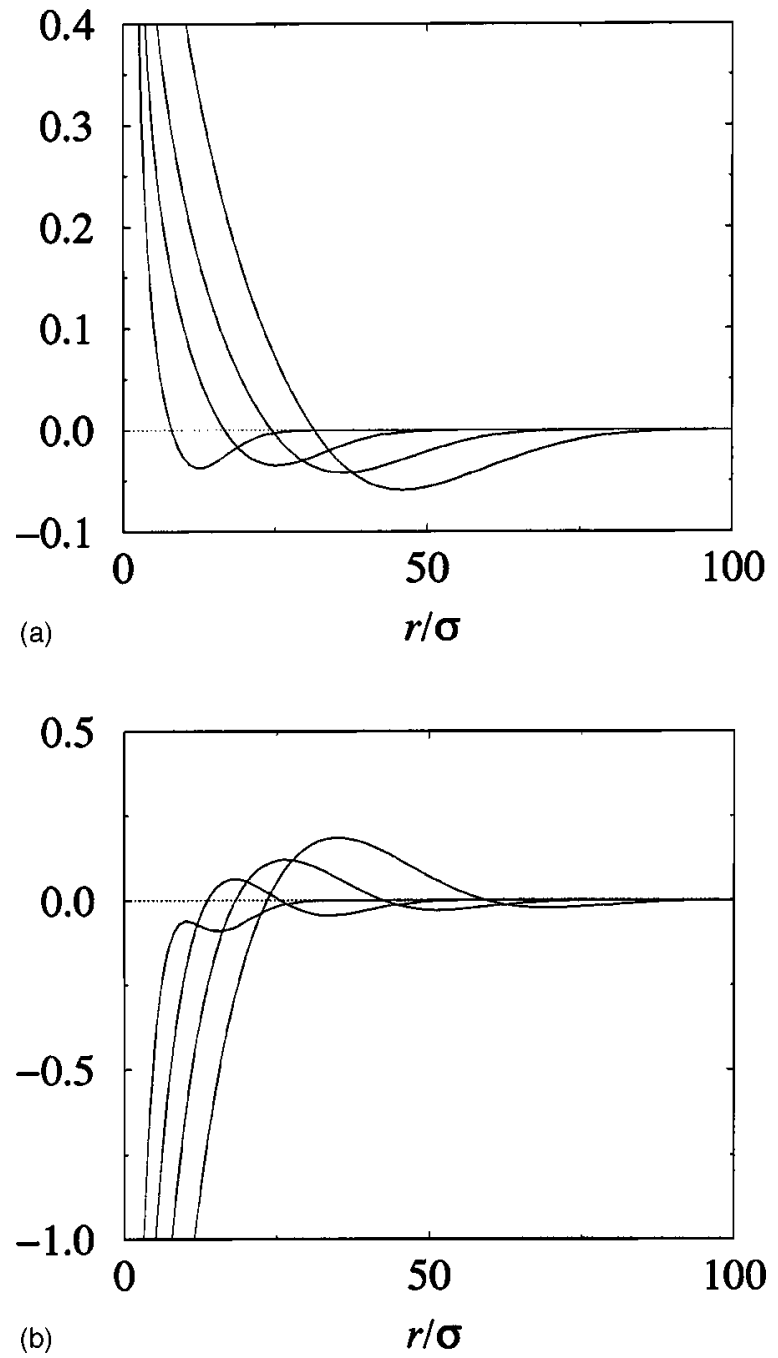

FIG. 11. Spatial correlation functions (a) $G_{n n}(r, t)$, in units $10^{-3} / \sigma^{4}$, and (b) $G_{n T}(r, t) / T(t)$, in units $10^{-3} / \sigma^{2}$, versus $r / \sigma$ obtained numerically from the structure factors shown in Fig. 7 at the same parameters as used in Fig. 5. Both functions show a growing correlation length.

We recall from Eqs. (A3) and (A4) that $\xi_{\|} \sim 1 / \gamma_{0}$ and $\xi_{H}$ $\sim \xi_{T} \sim 1 / \sqrt{\gamma_{0}}$, so that the behavior in Eqs. (42) and (43) is indeed on the expected scales. The function $g_{n}^{>}$does have the same qualitative shape as Fig. 11(a). The location of the minima also increases for large times as $\sqrt{\tau}$, but the minima of $g^{>}(x, s)$ are too shallow and too far to the right to give quantitative agreement with the asymptotic result (42). The behavior predicted by $g_{n}^{<}(x, s)$ is strictly positive, and describes the curves in Fig. 11(a) for $r$ to the right of the zero crossing, where $r \sim \xi_{H}$.

Comparison with simulation results confirms that the present theory correctly predicts the buildup of density correlations in the time regime $\tau<\tau_{c}$. For a more comprehensive comparison of the density correlation functions with MD simulations at different densities and different inelasticities, we refer to Ref. [4], where also the range of validity of the present theories is investigated.

In summary, the typical length scales of vortices $L_{\mathrm{v}}(t)$ $\sim \sqrt{\tau}$ and of density clusters $L_{\mathrm{cl}}(t) \sim \sqrt{\tau / \gamma_{0}}$, also correspond to the typical length scales on which the equal-time correla- 
tion functions $G_{\perp}(r, t), G_{\|}(r, t)$, and $G_{n n}(r, t)$ show structure, which extend far beyond the microscopic and kinetic scales.

\section{CONCLUSION}

In this paper the structure factors $S_{a b}(\mathbf{k}, t)$ and corresponding spatial correlation functions $G_{a b}(\mathbf{r}, t)$ have been calculated and compared with two-dimensional (2D) molecular dynamics simulations for weakly inelastic hard disk systems, where $\gamma_{0}=\left(1-\alpha^{2}\right) / 2 d$ is small, using the hydrodynamic equations of an elastic fluid, supplied with an energy sink representing the collisional dissipation. Also, we have assumed that the homogeneous cooling state (HCS) is an adiabatically cooling equilibrium state, which is only correct to lowest order in $\gamma_{0}$.

The basic theory is developed in Secs. III A, III B, and III C, modeled on the Cahn-Hilliard theory for spinodal decomposition. To understand the physical excitations that drive the instabilities, we have presented a theoretical analysis of the structure factors using a spectral analysis of the unstable Fourier modes. This is done in Sec. IV for structure factors and spatial correlations in the flow field, and in Sec. $\mathrm{V}$ for the density and temperature fields. The analysis gives a simple analytic description, valid for $\gamma_{0} \tau>1$, as given in Eqs. (29), (31), and (37).

It shows that the dynamics of the transverse and longitudinal flow fields on the largest length scales are controlled by two stable purely diffusive velocity fields with very different diffusivities. Agreement between the predictions of fluctuating hydrodynamics with Langevin noise for $S_{\|}$and $S_{\perp}$, and the results of MD simulations is very good, and holds far beyond the crossover time $\tau_{c}$, defined in Fig. 2. For instance, up to $\tau \simeq 100$ in Fig. 8 where $\tau_{c} \simeq 67$. Without the noise terms there is no quantitative agreement.

Calculation of the structure factor $S_{n n}(k, t)$ for the density fluctuations is essentially a linear stability analysis, which describes the early stages of clustering. It is expected to break down with increasing time, because the density fluctuations are predicted to grow at an exponential rate. This is indeed seen to happen as the time $\tau$ approaches the crossover time $\tau_{c}$ to the nonlinear clustering regime, as defined in the caption of Fig. 2. The agreement with MD simulations for $\tau<\tau_{c}$ is again good. Moreover, the simple analytic approximation (37), based on the dynamics of the unstable heat mode and valid for $\gamma_{0} \tau \gtrsim 2$, shows that the whole peak in $S_{n n}(k, t)$ lies in the $k$ range below the stability threshold for the heat mode $\left(k<k_{H}^{*}\right)$, and that the fluctuations with $k$ $>k_{H}^{*}$ are essentially at thermal noise level.

The density structure factor $S_{n n}(k, t)$ shows that spatial density fluctuations in undriven IHS fluids are unstable, and lead to the formation of density clusters. The linear instability is driven by longitudinal velocity fluctuations and described by a coupling coefficient of $\mathcal{O}\left(k^{2}\right)$ in Eqs. (37) and (38) for $S_{n n}$. The fluctuations in the flow field are only relatively unstable, and do not lead to exponential growth of the corresponding structure factors. Nevertheless, the dissipative IHS fluid develops structure on intermediate scales with typical length scales $L_{\mathrm{cl}}(t) \sim \xi_{\|} \sqrt{\gamma_{0} \tau} \sim \sqrt{\tau / \gamma_{0}}$ for the mean cluster sizes, and $L_{\mathrm{v}}(t) \sim \xi_{\perp} \sqrt{\gamma_{0} \tau} \sim \sqrt{\tau}$ for the mean vortex diameters.
Sections IV C and V B deal with spatial correlations. The assumption of incompressible flow for nearly elastic hard spheres $(\alpha \gtrsim 0.9)$ leads to spatial velocity correlations, including algebraic $r^{-d}$ tails, that are correct up to large distances $\left(r \lesssim 2 \pi \xi_{\|}\right)$. We have verified by MD simulations and numerical calculations that at small inelasticities $S_{\|}^{+}(k, t)$ essentially vanishes for all wave numbers except at very small$k$ values $\left(k \lesssim 1 / \xi_{\|}\right)$, where the assumption of incompressible u fluctuations, made in Sec. IV A, breaks down. Consequently, at small inelasticities the most important qualitative modification that $S_{\|}^{+}$adds to the spatial correlation function $G_{\|}(r, t)$ is to provide an exponential cutoff for the $r^{-d}$ tail at the largest scales $r \gtrsim 2 \pi \xi_{\|}$. At larger inelasticities the nonvanishing contributions from $S_{\|}^{+}(k, t)$ modify $G_{\|}(r, t)$ and $G_{\perp}(r, t)$ significantly at all distances.

The good quantitative agreement between theory and computer simulations shows that our theory for structure factors, $S_{\alpha \beta}(\mathbf{k}, t)$ and $S_{n n}(k, t)$, and spatial correlation functions, $G_{\alpha \beta}(\mathbf{r}, t)$ and $G_{n n}(r, t)$, is correct for wave number, position, and time dependence in the relevant hydrodynamic range and for inelasticities ( $\alpha \gtrsim 0.6)$ that are not too large.

\section{ACKNOWLEDGMENTS}

The authors want to thank R. Brito for many clarifying discussions, and his help in deriving the results of Sec. V. We also thank R. Brito and J.A.G. Orza for a pleasant collaboration and for making their unpublished simulation results available to us. Thanks are also due to W. van de Water for pointing out that relation (B7) is well known in the theory of homogeneous turbulence. M.E. wants to thank E. Ernst for providing the Mount Uluru analog. Furthermore, M.E. acknowledges financial support from the Office of International Relations of Universidad Complutense in Madrid, where this paper was completed. T.v.N. acknowledges support of the foundation 'Fundamenteel Onderzoek der Materie (FOM)," which is financially supported by the Dutch National Science Foundation (NWO).

\section{APPENDIX A: TRANSPORT COEFFICIENTS}

Linearization of the hydrodynamic equations (1) around the HCS results in the following set of equations:

$$
\begin{gathered}
\partial_{t} \delta n=-n \boldsymbol{\nabla} \cdot \mathbf{u}, \\
\partial_{t} \mathbf{u}=-\frac{1}{\rho} \boldsymbol{\nabla} p+\nu \nabla^{2} \mathbf{u}+\left(\nu_{l}-\nu\right) \nabla \boldsymbol{\nabla} \cdot \mathbf{u}, \\
\partial_{t} \delta T=\frac{2 \kappa}{d n} \nabla^{2} \delta T-\frac{2 p}{d n} \nabla \cdot \mathbf{u}-\delta \Gamma .
\end{gathered}
$$

The pressure $p$ is assumed to be that of elastic hard spheres (EHS), $p=n T\left(1+\Omega_{d} \chi n \sigma^{d} / 2 d\right)$, where $\Omega_{d}=2 \pi^{d / 2} / \Gamma(d / 2)$ is the $d$-dimensional solid angle, and $\chi(n)$ is the equilibrium value of the pair correlation function of EHS of diameter $\sigma$ at contact. In three dimensions the Carnahan-Starling approximation gives $\chi=(2-\phi) / 2(1-\phi)^{3}$ [35], in two dimensions the Verlet-Levesque approximation gives $\chi=(1$ $-7 \phi / 16) /(1-\phi)^{2}[36]$. 
The transport coefficient $\nu, \nu_{l}$, and $\kappa$ for hard disks and hard spheres are given by the Enskog theory for elastic spheres [28], and $\rho \nu=\eta$ and $\rho \nu_{l}=2 \eta(1-1 / d)+\zeta$ are expressed in shear and bulk viscosity $\eta$ and $\zeta$, respectively. The collision frequency in the Enskog theory is $\omega$ $=\Omega_{d} \chi n \sigma^{d-1} \mathrm{v}_{0} / \sqrt{2 \pi}$ where the thermal velocity $\mathrm{v}_{0}$ $=\sqrt{2 T / m}$, and temperature is measured in units of Boltzmann's constant $k_{B}$.

Carrying out the linearization and rescaling described above Eq. (3) yields the hydrodynamic matrix

$$
\mathbf{M}(\mathbf{k})=\left(\begin{array}{cccc}
0 & 0 & -i k l_{0} & 0 \\
-\gamma_{0} a & \beta(k) & -i b k l_{0} & 0 \\
-i c_{T}^{2} k l_{0} & -\frac{d}{4} i b k l_{0} & \delta_{l}(k) & 0 \\
0 & 0 & 0 & \delta_{\perp}(k)
\end{array}\right)
$$

with transport coefficient and correlation lengths $\xi_{a}$,

$$
\begin{gathered}
\delta_{\perp}=\gamma_{0}-\nu k^{2} / \omega \equiv \gamma_{0}\left(1-k^{2} \xi_{\perp}^{2}\right), \\
\delta_{l}=\gamma_{0}-\nu_{l} k^{2} / \omega \equiv \gamma_{0}\left(1-k^{2} \xi_{l}^{2}\right), \\
\beta=-\gamma_{0}-2 \kappa k^{2} / d n \omega \equiv-\gamma_{0}\left(1+k^{2} \xi_{T}^{2}\right) .
\end{gathered}
$$

Moreover, we need the correlation lengths $\xi_{H}=\xi_{T}\left(c_{T} / c_{s}\right)$ and $\xi_{\|}$, with

$$
\xi_{\|}^{2}=\xi_{l}^{2}+\left(l_{0} / \gamma_{0}\right)^{2}\left[c_{T}^{2}+\frac{1}{8} d b(b-a)\right]
$$

to be derived below. Note that all $\xi_{a} \sim 1 / \sqrt{\gamma_{0}}$ except $\xi_{\|}$ $\sim 1 / \gamma_{0}$, which is much larger.

The thermodynamic quantities are

$$
\begin{gathered}
a=2\left[1+\frac{n}{\chi} \frac{d \chi}{d n}\right], \quad c_{T}^{2}=\frac{\mathrm{v}_{T}^{2}}{\mathrm{v}_{0}^{2}}=\frac{1}{2 T}\left(\frac{\partial p}{\partial n}\right)_{T}, \\
b=\frac{2 p}{d n T}, \quad c_{s}^{2}=\frac{\mathrm{v}_{s}^{2}}{\mathrm{v}_{0}^{2}}=c_{T}^{2}+\frac{d}{4} b^{2},
\end{gathered}
$$

where $\mathbf{v}_{T}$ and $\mathbf{v}_{s}$ are, respectively, the isothermal and adiabatic speed of sound for EHS fluids.

The eigenvalues of the asymmetric matrix $\mathbf{M}(\mathbf{k})$ are denoted by $\zeta_{\lambda}(\mathbf{k})$ and its right and left eigenvectors by $\mathbf{w}_{\lambda}(\mathbf{k}), \mathbf{v}_{\lambda}(\mathbf{k})$ respectively. Here $\lambda= \pm$ labels the sound modes, $\lambda=H$ the heat mode, and $\lambda=\perp$ labels $(d-1)$ degenerate shear or transverse velocity modes. The eigenvectors form a complete biorthonormal basis, which satisfies $\left\langle\mathbf{v}_{\lambda} \mid \mathbf{w}_{\mu}\right\rangle=\delta_{\lambda \mu}$, and allows a spectral decomposition of $\mathbf{M}(\mathbf{k})$ in the form

$$
\mathbf{M}(\mathbf{k})=\sum_{\lambda}\left|\mathbf{w}_{\lambda}(\mathbf{k})\right\rangle \zeta_{\lambda}(k)\left\langle\mathbf{v}_{\lambda}(\mathbf{k})\right|
$$

Moreover, the eigenvalue equation, $\operatorname{det}[\zeta(k) \mathbf{I}-\mathbf{M}(\mathbf{k})]=0$, is an even function of $\mathbf{k}$. Consequently, $\mathbf{M}(\mathbf{k})$ and $\mathbf{M}(-\mathbf{k})$ have the same eigenvalues, which are either real or form a complex conjugate pair. So we choose $\zeta_{\lambda}(\mathbf{k})=\zeta_{\lambda}(-\mathbf{k})$ $=\zeta_{\lambda}(k)$. The corresponding eigenvectors of $\mathbf{M}(-\mathbf{k})$ in the case of propagating sound modes are obtained from the transformation $\left\{\mathbf{w}_{+}(-\mathbf{k}), \mathbf{v}_{-}(-\mathbf{k})\right\} \leftrightarrow\left\{\mathbf{w}_{-}(\mathbf{k}), \mathbf{v}_{+}(\mathbf{k})\right\}$. All other eigenvectors, corresponding to nonpropagating modes, are invariant under the transformation $\mathbf{k} \rightarrow-\mathbf{k}$.

Inspection of Eq. (A2) gives for the shear modes $\zeta_{\perp}(k)$ $=\gamma_{0}\left(1-k^{2} \xi_{\perp}^{2}\right)$ and $\mathbf{w}_{\perp}(\mathbf{k})=\mathbf{v}_{\perp}(\mathbf{k})=\widetilde{\mathbf{u}}_{\perp}(\mathbf{k})$. This mode is unstable, i.e., has a positive growth rate $\zeta_{\perp}(k)$ for $k$ below the stability threshold $k_{\perp}^{*}=1 / \xi_{\perp} \sim \sqrt{\gamma_{0}} / l_{0}$.

The eigenvalues of the remaining $3 \times 3$ matrix are the roots of a cubic equation. They are determined numerically and shown in Fig. 3. The characteristic properties of the spectrum and eigenmodes are discussed in the body of the paper after Eq. (3). The more technical aspects needed in the calculations are summarized below.

There is a second unstable mode, the heat mode with $\zeta_{\lambda}$ $>0$ for $k<k_{H}^{*}$. This stability threshold is the root of $\zeta_{H}(k)$ $=0$ or equivalently of $\operatorname{det}|\mathbf{M}(\mathbf{k})|=0$, and yields simply

$$
k_{H}^{*}=\frac{1}{\xi_{T}}\left(\frac{d a b}{4 c_{T}^{2}}-1\right)^{1 / 2} .
$$

The three remaining eigenvalues and eigenvectors can be calculated perturbatively. In the dissipative range $\left(k l_{0} \ll \gamma_{0}\right)$ this is done by an expansion in powers of $k$, and yields after lengthy but straightforward calculations, the eigenvalue $\zeta_{H}$ $=\gamma_{0}\left(1-k^{2} \xi_{\|}^{2}\right)$ [see Eq. (4)], and the corresponding left and right eigenvectors, where components are labeled as $(n, T, l)$,

$$
\begin{gathered}
\mathbf{w}_{H}(\mathbf{k})=\left(-\frac{i k l_{0}}{\gamma_{0}}, \frac{i k l_{0}}{2 \gamma_{0}}(a-b), 1\right), \\
\mathbf{v}_{H}(\mathbf{k})=\left(-\frac{i k}{\gamma_{0}}\left(\frac{d}{8} a b-c_{T}^{2}\right),-\frac{i k d b}{8}, 1\right)
\end{gathered}
$$

with normalization $\left\langle\mathbf{v}_{H} \mid \mathbf{w}_{H}\right\rangle=1+\mathcal{O}\left(k^{2}\right)$. The "sound" modes are stable for all wavelengths, and nonpropagating in this $k$ range. Inspection of Fig. 3 shows that the mode $(\lambda=$ $+)$ decays diffusively $\left(\zeta_{+} \propto-k^{2}\right)$, whereas the mode $(\lambda=$ $-)$ decays rapidly $\left(\zeta_{-} \propto-\gamma_{0}\right)$. The explicit forms of $\zeta_{+}$and $\zeta_{-}$are not needed here. They can be found in [37]. The corresponding eigenmodes are only needed to lowest nonvanishing order in $k$ and read

$$
\begin{gathered}
\mathbf{w}_{+}(\mathbf{k})=(1,-a, 0), \quad \mathbf{w}_{-}=(0,1,0), \\
\mathbf{v}_{+}(\mathbf{k})=(1,0,0), \quad \mathbf{v}_{-}=(a, 1,0) .
\end{gathered}
$$

In the dissipative range, as $k \rightarrow 0$, the heat mode (A8) is a purely longitudinal velocity fluctuation, while the sound modes (A9) are a mixture of density and temperature fluctuations. To first order in $k$, density and temperature fluctuations couple to the heat mode, and longitudinal velocity fluctuations couple to the sound modes.

For larger wave numbers $\left(k l_{0} \sim \sqrt{\gamma_{0}}\right)$ the conventional character of the sound and the heat modes is recovered. Here we solve the eigenvalue problem by setting $k=\sqrt{\gamma_{0}} q$, taking $q=\mathcal{O}(1)$, and determine the eigenvalues and eigenvectors to 
dominant order as $\gamma_{0} \rightarrow 0$ (nearly elastic case). The eigenvalue for the heat mode in the elastic range is then

$$
\zeta_{H}(k)=\gamma_{0} \xi_{H}^{2}\left(k_{H}^{* 2}-k^{2}\right) \equiv \gamma_{0}\left(\zeta_{0}-k^{2} \xi_{H}^{2}\right),
$$

as listed in Eq. (4), and $\omega \gamma_{0} \xi_{H}^{2}=D_{T}$ is the heat diffusivity of the elastic hard sphere fluid. The corresponding eigenvectors are

$$
\begin{aligned}
& \mathbf{w}_{H}(\mathbf{k})=\frac{1}{c_{s}}\left(\frac{d}{4} b,-c_{T}^{2},-d b \zeta_{H}(k) / 4 i k\right), \\
& \mathbf{v}_{H}(\mathbf{k})=\frac{1}{c_{s}}\left(b,-1,\left[a \gamma_{0}-b \zeta_{H}(k)\right] / i k c_{T}^{2}\right) .
\end{aligned}
$$

In the elastic limit $\left(\gamma_{0} \rightarrow 0\right)$ the components $w_{H l} \sim \mathrm{v}_{\mathrm{Hl}}$ $\sim \sqrt{\gamma_{0}}$ are vanishing, and the surviving terms in Eq. (A11) give the heat mode for the elastic fluid.

For the sound mode $(\lambda=\sigma= \pm)$ in the elastic $k$ range we have similarly $\zeta_{H}=-i \sigma c_{s} k l_{0}-\mathcal{O}\left(k^{2}\right)$, and the eigenvectors are those of the elastic fluid,

$$
\begin{gathered}
\mathbf{w}_{\sigma}(\mathbf{k})=\frac{1}{c_{s}}\left(1, b, \sigma c_{s}\right), \\
\mathbf{v}_{\sigma}(\mathbf{k})=\frac{1}{2 c_{s}}\left(c_{T}^{2}, \frac{d}{4} b, \sigma c_{s}\right) .
\end{gathered}
$$

\section{APPENDIX B: FOURIER TRANSFORMS}

To calculate the tensor velocity correlation function $G_{\alpha \beta}(\mathbf{r}, t)$ by Fourier inversion from $S_{\alpha \beta}(\mathbf{k}, t)$, we start from Eqs. (24)-(27), and consider first the incompressible limit where $S_{\|}(k, t)=0$, i.e.,

$$
G_{\alpha \beta}^{+}(\mathbf{r}, t)=\int \frac{d \mathbf{k}}{(2 \pi)^{d}} e^{i \mathbf{k} \cdot \mathbf{r}}\left(\delta_{\alpha \beta}-\hat{k}_{\alpha} \hat{k}_{\beta}\right) S_{\perp}^{+}(k, t) .
$$

According to Eq. (25) $G_{\alpha \beta}$ can be split into two scalar functions, $G_{\|}$and $G_{\perp}$, which will be expressed in $S_{\perp}$. The simplest functions to calculate are the trace and parallel part of $G_{\alpha \beta}$, i.e.,

$$
\begin{aligned}
\frac{G_{p p}^{+}(r, t)}{d-1} & =\sum_{\alpha} \frac{G_{\alpha \alpha}^{+}(\mathbf{r}, t)}{d-1}=\int \frac{d \mathbf{k}}{(2 \pi)^{d}} e^{i \mathbf{k} \cdot \mathbf{r}^{+}} S_{\perp}^{+}(k, t), \\
G_{\|}^{+}(r, t) & =\hat{r}_{\alpha} \hat{r}_{\beta} G_{\alpha \beta}^{+}(\mathbf{r}, t) \\
& =\int \frac{d \mathbf{k}}{(2 \pi)^{d}} e^{i \mathbf{k} \cdot \mathbf{r}}\left[1-(\hat{\mathbf{k}} \cdot \hat{\mathbf{r}})^{2}\right] S_{\perp}^{+}(k, t) .
\end{aligned}
$$

To carry out the $d$-dimensional angular integrations for $d$ $\geqslant 2$ we express the infinitesimal solid angle as

$$
d \hat{\mathbf{k}}=\left(\sin \theta_{1}\right)^{d-2} \cdots\left(\sin \theta_{d-2}\right) d \theta_{1} \cdots d \theta_{d-2} d \phi
$$

where $\theta_{n} \in(0, \pi)$ are polar angles and $\phi \in(0,2 \pi)$ is an azimuthal angle, and we note that the full solid angle is $\Omega_{d}$ $=\int d \hat{\mathbf{k}}=2 \pi^{d / 2} / \Gamma(d / 2)$. Then we use the relation

$$
\begin{aligned}
\int \frac{d \hat{\mathbf{k}}}{\Omega_{d}} \exp (i \mathbf{k} \cdot \mathbf{r}) & =\frac{\int_{0}^{\pi} d \theta(\sin \theta)^{d-2} \exp (i k r \cos \theta)}{\int_{0}^{\pi} d \theta(\sin \theta)^{d-2}} \\
& =\left(\frac{2}{k r}\right)^{d / 2-1} \Gamma(d / 2) J_{d / 2-1}(k r),
\end{aligned}
$$

where the integral representation (8.411.7) of Ref. [38] has been used for the Bessel function $J_{v}(z)$. Then Eqs. (B2) become

$$
\begin{gathered}
G_{p p}^{+}(r, t)=\frac{d-1}{(2 \pi)^{d / 2} r^{d / 2-1}} \int_{0}^{\infty} d k k^{d / 2} J_{d / 2-1}(k r) S_{\perp}^{+}(k, t), \\
G_{\|}^{+}(r, t)=\frac{d-1}{(2 \pi r)^{d / 2}} \int_{0}^{\infty} d k k^{d / 2-1} J_{d / 2}(k r) S_{\perp}^{+}(k, t) .
\end{gathered}
$$

With the help of the recursion formula for Bessel functions, $z d J_{\nu}(z) / d z+\nu J_{\nu}(z)=z J_{\nu-1}(z)$, together with the general relation

$$
G_{\perp}^{+}(r, t)=\left[G_{p p}^{+}(r, t)-G_{\|}^{+}(r, t)\right] /(d-1),
$$

we obtain $G_{\perp}^{+}(r, t)$ from $G_{\|}^{+}(r, t)$, i.e.,

$$
G_{\perp}^{+}(r, t)=G_{\|}^{+}(r, t)+\left(\frac{r}{d-1}\right) \frac{\partial}{\partial r} G_{\|}^{+}(r, t) .
$$

In the general case $S_{\|}(k, t)$ is nonvanishing and we have from Eq. (24) an additional part, denoted by $\bar{G}_{\alpha \beta}(r, t)$, coming from $\hat{k}_{\alpha} \hat{k}_{\beta} S_{\|}(k, t)$. Here we have the relations

$$
\begin{gathered}
\bar{G}_{\perp}^{+}(r, t)=\int \frac{d \mathbf{k}}{(2 \pi)^{d}} e^{i \mathbf{k} \cdot \mathbf{r}} \frac{\left[1-(\hat{\mathbf{k}} \cdot \hat{\mathbf{r}})^{2}\right] S_{\|}^{+}(k, t)}{d-1}, \\
\bar{G}_{p p}^{+}(r, t)=\int \frac{d \mathbf{k}}{(2 \pi)^{d}} e^{i \mathbf{k} \cdot \mathbf{r}} S_{\|}^{+}(k, t) .
\end{gathered}
$$

The results for these functions can be read off directly from Eq. (B2) and (B5). In this case the parallel part is obtained from Eq. (B8) as

$$
\begin{aligned}
\bar{G}_{\|}^{+}(r, t)= & \bar{G}_{p p}^{+}(r, t)-(d-1) \bar{G}_{\perp}^{+}(r, t)=\bar{G}_{\perp}^{+}(r, t) \\
& +r \frac{\partial}{\partial r} \bar{G}_{\perp}^{+}(r, t) .
\end{aligned}
$$

Fourier inversion of any of the scalar functions $S_{a b}(k, t)$ with $a, b=\{n, T\}$ is covered by the first line of Eqs. (B2) and (B5). We consider first $G_{\|}$and $G_{\perp}$ in the incompressible limit, where $S_{\perp}(k, t)$ is given by Eq. (29).

Inspection of Eq. (29) shows that the large- $k$ limit of $S_{\perp}(k, t)$ is $S_{\perp}^{\infty}=T(t) / \rho$, leaving $S_{\perp}^{+}(k, t)$ as a remainder. This may be written as

$$
S_{\perp}^{+}(k, t)=\frac{T(t)}{\rho} \int_{0}^{2 \gamma_{0} \tau} d s^{\prime} \exp \left[\left(1-k^{2} \xi_{\perp}^{2}\right) s^{\prime}\right] .
$$


Using Eq. (B5) for the parallel velocity correlation function $G_{\|}^{+}(r, t)$ and Eq. (B7) to determine $G_{\perp}^{+}(r, t)$, we obtain $G_{\lambda}^{+}(r, t)=\left[T(t) / \rho \xi_{\perp}^{d}\right] g_{\lambda}(x, s)$ for $\lambda=\{\|, \perp\}$ with $s=2 \gamma_{0} \tau$ and $x=r / \xi_{\perp}$, which is valid in dimensions $d \geqslant 2$. Moreover, $g_{\lambda}(x, s)$ is given by

$$
\begin{aligned}
& g_{\perp}(x, s)=\int_{0}^{s} d s^{\prime} \frac{e^{s^{\prime}-x^{2} / 4 s^{\prime}}}{\left(4 \pi s^{\prime}\right)^{d / 2}}-\frac{g_{\|}(x, s)}{d-1}, \\
& g_{\|}(x, s)=\frac{d-1}{2 \pi^{d / 2} x^{d}} \int_{0}^{s} d s^{\prime} e^{s^{\prime}} \gamma\left(\frac{d}{2}, \frac{x^{2}}{4 s^{\prime}}\right) .
\end{aligned}
$$

The Bessel transform Eq. (B5) of $\exp \left(-\beta k^{2}\right)$ in Eq. (B10) has been calculated using Eq. (6.631.5) of Ref. [38], where $\gamma(\alpha, z)=\int_{0}^{z} d t \exp (-t) t^{\alpha-1}$ is the incomplete gamma function. For $d=2$ it reduces to $\gamma(1, z)=1-\exp (-z)$ and for $d$ $=3$ to $\gamma(3 / 2, z)=\sqrt{\pi} \phi(\sqrt{z}) / 2-\sqrt{z} \exp (-z)$, where $\phi(z)$ is the error function. We observe that $g_{\|}(x, s)$ is positive for all $x, s$. For large distance, $x^{2} \gg 4 s$, the functions $g_{\lambda}(x, s)$ show algebraic tails $\sim 1 / x^{d}$. This can be seen by noting that $\gamma\left(\alpha, x^{2} / 4 s\right)$ approaches $\Gamma(\alpha)$, so that $g_{\|} \simeq-(d-1) g_{\perp}$ and

$$
\frac{g_{\|}(x, s)}{d-1}=-g_{\perp}\left(x_{\perp}, s\right) \sim\left(\frac{1}{\Omega_{d} x^{d}}\right)\left[e^{s}-1\right] .
$$

[1] T.P.C. van Noije, M.H. Ernst, R. Brito, and J.A.G. Orza, Phys. Rev. Lett. 79, 411 (1997).

[2] T.P.C. van Noije, R. Brito, and M.H. Ernst, Phys. Rev. E 57, R4891 (1998).

[3] T.P.C. van Noije, Ph.D. thesis, Universiteit Utrecht, 1999.

[4] J.A.G. Orza, R. Brito, and M.H. Ernst (unpublished).

[5] J.S. Langer, in Solids Far from Equilibrium, edited C. Godrèche (Cambridge University Press, Cambridge, 1992).

[6] Holmes' Principles of Physical Geology, edited by D. Duff (Chapman and Hall, Boca Raton, FL, 1994), p. 368.

[7] T.P.C. van Noije, M.H. Ernst, E. Trizac, and I. Pagonabarraga, Phys. Rev. E 59, 4326 (1999).

[8] G. Grinstein, D.-H. Lee, and S. Sachdev, Phys. Rev. Lett. 64, 1927 (1990); G. Grinstein, J. Appl. Phys. 69, 5441 (1991).

[9] S.F. Edward and D.R. Wilkinson, Proc. R. Soc. London, Ser. A 381, 17 (1982).

[10] I. Goldhirsch and G. Zanetti, Phys. Rev. Lett. 70, 1619 (1993).

[11] I. Goldhirsch, M-L. Tan, and G. Zanetti, J. Sci. Comput. 8, 1 (1993).

[12] S. McNamara, Phys. Fluids A 5, 3056 (1993).

[13] S.E. Esipov and T. Pöschel, J. Stat. Phys. 86, 1385 (1997).

[14] J.J. Brey, F. Moreno, and A. Santos, Phys. Rev. E 54, 445 (1996).

[15] P. Deltour, and J.-L. Barrat, J. Phys. I 7, 137 (1997).

[16] J.J. Brey, F. Moreno, and M.J. Ruiz-Montero, Phys. Fluids 10, 2965 (1998); 10, 2976 (1998).

[17] R. Brito, and M.H. Ernst, Europhys. Lett. 43, 497 (1998); Int. J. Mod. Phys. C 9, 1339 (1998).

[18] S. Luding, and H.J. Herrmann, Chaos 9, 25 (1999).

[19] L. Landau and E.M. Lifshitz, Fluid Mechanics (Pergamon Press, New York, 1959).

[20] U. Frisch, Turbulence: The Legacy of A.N. Kolmogorov (Cam- bridge University Press, Cambridge, 1996).

[21] D. Rothman, J. Stat. Phys. 56, 517 (1989).

[22] J.J. Brey M.J. Ruiz-Montero, and D. Cubero, Phys. Rev. E 60, 3150 (1999).

[23] P.K. Haff, J. Fluid Mech. 134, 401 (1983).

[24] C.K.K. Lun, S.B. Savage, D.J. Jeffrey, and N. Chepurniy, J. Fluid Mech. 140, 223 (1984).

[25] J.T. Jenkins and M.W. Richman, Arch. Ration. Mech. Anal. 87, 355 (1985).

[26] A. Goldshtein and M. Shapiro, J. Fluid Mech. 282, 75 (1995).

[27] T.P.C. van Noije and M.H. Ernst, Granular Matter 1, 57 (1998).

[28] S. Chapman and T.G. Cowling, The Mathematical Theory of Non-uniform Gases (Cambridge University Press, Cambridge, England, 1970).

[29] S. Chen, Y. Deng, X. Nie, and Y. Tu, e-print cond-mat/9804235.

[30] E. Ben-Naim, S.Y. Chen, G.D. Doolen, and S. Redner, e-print cond-mat/9909354.

[31] N.V. Brilliantov and T. Pöschel, e-print cond-mat/9906404.

[32] I. Goldhirsch and T.P.C. van Noije, Phys. Rev. E ( to be published).

[33] G.K. Batchelor, The Theory of Homogeneous Turbulence (Cambridge University Press, Cambridge, 1970), Chap. 3.

[34] J.R. Dorfman, T.R. Kirkpatrick, and J.V. Sengers, Annu. Rev. Phys. Chem. 45, 213 (1994).

[35] N.F. Carnahan and K.E. Starling, J. Chem. Phys. 51, 635 (1969).

[36] L. Verlet and D. Levesque, Mol. Phys. 46, 969 (1982).

[37] http://seneca.fis.ucm.es/brito/IHS.html

[38] I.S. Gradshteyn and I.M. Ryzhik, Table of Integrals, Series, and Products (Academic Press, New York, 1965). 